\title{
Interspecies Evolution and Networks Investigation of the Auxin Response Protein (AUX/IAA) Family Reveals the Adaptation Mechanisms of Halophytes Crops in Nitrogen Starvation Agroecological Environments
}

\author{
Haomiao Yu (D), Yuan Yuan, Sijiao Wang, Guoming Wu, Haishen Xu, Jianglan Wei, Le Ju, Yulin Huang \\ and Hui Chen *D \\ College of Life Science, Sichuan Agricultural University, Ya'an 625014, China; smkxyuhaomiao@163.com (H.Y.); \\ yuan1042001489@163.com (Y.Y.); scnydxwangsijiao@163.com (S.W.); wuguomingsmkx@163.com (G.W.); \\ haishenxu27g@163.com (H.X.); weijianglan2021@163.com (J.W.); scnydxjule@163.com (L.J.); \\ sicauhuangyulin@163.com (Y.H.) \\ * Correspondence: chenhui@sicau.edu.cn
}

Citation: Yu, H.; Yuan, Y.; Wang, S.; Wu, G.; Xu, H.; Wei, J.; Ju, L.; Huang, Y.; Chen, H. Interspecies Evolution and Networks Investigation of the Auxin Response Protein (AUX/IAA) Family Reveals the Adaptation Mechanisms of Halophytes Crops in Nitrogen Starvation Agroecological Environments. Agriculture 2021, 11, 780. https://doi.org/10.3390/ agriculture 11080780

Academic Editor: Sergio Esposito

Received: 28 July 2021

Accepted: 13 August 2021

Published: 16 August 2021

Publisher's Note: MDPI stays neutra with regard to jurisdictional claims in published maps and institutional affiliations.

Copyright: (c) 2021 by the authors. Licensee MDPI, Basel, Switzerland. This article is an open access article distributed under the terms and conditions of the Creative Commons Attribution (CC BY) license (https:/ / creativecommons.org/licenses/by/ $4.0 /)$
Abstract: The maintenance of adaptability to the exposure to agroecological extreme environments is generally a feature after the long-term domestication of crops. Auxin influences plant growth in all environments. At present, the research on the auxin response factors ( $A R F s)$ has been very in-depth. However, there is still a large gap in the research on the origin, evolution, and regulatory networks of the Auxin-responsive protein (AUX/IAA) family. Here, we identified 495 AUX/IAAs from 19 representative species covering aquatic algae to angiosperms and found that they originated from early bryophytes and mainly expanded by polyploidy in angiosperms. In the domesticated crop quinoa, the evolutionary model of the IAA family is relatively independent and forms a robust regulatory network with auxin signals and energy metabolism pathways. In the nitrogen-deficient environment, CqIAAs (Chenopodium quinoa AUX/IAAs), auxin signals, and TCA pathway genes induced expression in young roots to promote root elongation and could regulate the balance of carbon and nitrogen metabolism to maintain the adaptation of early seedlings in poor environments. Furthermore, a rapidly evolving CqIAA (AUR62011942) not only has different expression levels in two quinoa seeds but also has a significant stress response when seedlings face nitrogen deficiency stress, which may be a key factor in the adaptive regulation of the barren environment. Our research provides valuable clues for understanding the origin, evolution, and functional innovation of auxin signaling and also provides a reference for future agricultural breeding in the context of global environmental changes.

Keywords: AUX/IAA family; plant population; quinoa; nitrogen starvation; regulatory networks; system evolution

\section{Introduction}

In the process of plant evolution from water to land, it experienced complex and violent environmental changes and formed the survival mechanism regulated by various complex environmental signals through singular evolutionary strategies [1]. How land plants adapt and respond to harsh stress without water-surrounded environments is an intriguing and fundamental biological question. Moreover, improving plant resistance is essential for agricultural productivity and environmental sustainability. To survive in a variety of environmental stresses, plants regulate multiple metabolic pathways and have evolved a variety of adaptive characteristics governed by complex signals and regulatory systems, as well as downstream networks controlled by transcription factors (TFs) [2,3]. The auxin signaling pathway is involved in the most basic biological process of plant growth and development, which determines the fate of plants from birth to death $[4,5]$. In 
an intact auxin signaling pathway, transport inhibitor response (TIR) proteins sense auxin and bind to it, further sending downstream cascading signals that promote the interaction between Auxin response factor (ARF) and AUX/IAA, and triggering ubiquitin-mediated AUX/IAA protein degradation through the SCF ${ }^{\mathrm{TIR} 1 / \mathrm{AFB}}$ complex [6,7]. Aux/IAA protein has no DNA-binding domain and relies on the binding with ARF protein. Through the shared C-terminal domain, ARF-IAA dimers can physically interact with the target gene to regulate its transcriptional activity [8]. The structure, function, and evolution of ARF have been widely and deeply reported [9-11]. Regrettably, there is no systematic analysis on the origin, evolution, and function of its interaction protein AUX/IAA family so far.

Quinoa (Chenopodium quinoa Willd. $2 \mathrm{n}=4 \mathrm{x}=36$ ) is an allotetraploid crop of which cultivation and domestication began over 7000 years ago and originated in the Andes of South America as the Incans' primary food source [12]. Quinoa has greater adaptation ability to high altitude, strong light, cold environments, and especially poor soils, because of its low nutrient requirements [13]. Carbohydrates and saccharides are the most valuable types of energy and nutrition sources in crops, and up to 58.1-64.2\% of quinoa content may be the energy and nutritional reserves to respond to agroecological extremes [14]. Because of the selection and domestication by generations of farmers, quinoa has a rich genetic diversity [12]. As a typical polyploid crop, it provides an ideal material for studying preferential gene retention after polyploidization. Whether transcription factors with rich basic functions leave a genomic imprint in domesticated plants after a long period of human domestication is an open question. Complete genetic research frameworks were not developed until the genome sequence was published in 2017 [15], which provided strong support for excavating the complex metabolic pathways and transcriptional regulatory factors of quinoa.

Herein, we comprehensively identified the expansion and evolutionary origin of the IAA family from 19 genomes including Arabidopsis thaliana, Oryza sativa, Amborella trichopoda, the representative polyploid Chenopodium quinoa, and its related species. Next, we found that the widespread expansion of AUX/IAAs in quinoa and other species was associated with polyploidy. Genome synteny revealed that AUX/IAAs' evolution patterns in quinoa are relatively independent and are without strongly collinearity relationship with other angiosperms. More importantly, we found that polyploid-related CqIAAs may be an integral part of hormone signaling and energy metabolism pathways, adapting quinoa to the environment by regulating hormone signaling and maintaining carbon-nitrogen metabolic balance in changing environments. It is worth noting that we have identified a positive selection gene, which is highly expressed in quinoa seedlings under nitrogendeficient conditions, and it may play an important role in maintaining the balance of nitrogen-deficiency in plants. Our study provides valuable clues for understanding the evolutionary history of IAA family from aquatic plants to higher angiosperms and also proposes an evolutionary strategy for crop adaptation to poor environment, thus providing a blueprint for future breeding of superior strains with poor environment adaptability.

\section{Materials and Methods}

\subsection{Plant Materials and Growth Conditions}

The plant materials we used in this paper, pale-wheat (Qing-Baili) and dark-yellow (Qing-Huangli) C. quinoa variety, were obtained from the Gao Yuan Jinhe Ecological Agriculture and Animal Husbandry Technology Co., Ltd. (Qinghai, China). The fruit weight and fruit size of Qing-Baili are significantly higher than Qing-Huangli, which is just at the core of our study. In this paper, we describe Qing-baili as large-fruit quinoa (LF quinoa), and we describe Qing-huangli as small fruit quinoa (SF quinoa).

The quinoa seeds were soaked in sterile water and germinated at $25{ }^{\circ} \mathrm{C}$ and then transplanted into the hydroponic boxes when two cotyledons fully unfolded. The hydroponic materials were cultured in the plant culture room of the College of Life Sciences of Sichuan Agricultural University under $22{ }^{\circ} \mathrm{C}$, alternately given $12 \mathrm{~h}$ of light and $8 \mathrm{~h}$ of darkness. The white light intensity was $100 \mu \mathrm{mol} \mathrm{m} \mathrm{m}^{-2} \mathrm{~s}^{-1}$, and the wavelengths were 
mainly from 400 to $510 \mathrm{~nm}$ and from 610 to $720 \mathrm{~nm}$. The hydroponic conditions were set as two groups: the control group was treated with Hoagland complete nutrient solution, and the nitrogen-deficient Hoagland formula was used in the nitrogen-deficient treatment group. We replaced the culture medium every three days. Two weeks after the growth of seedlings, the roots and leaves of quinoa were collected and stored in a refrigerator at $-80^{\circ} \mathrm{C}$ for subsequent physiological and qPCR experiments.

2.2. Orthologous Inference, Phylogenetic, Structural Analysis of AUX/IAA Family in Multiple Species and Identification of Auxin, Triarboxylic Acid Cycle Pathway Genes in Quinoa

The Arabidopsis genome data were obtained from the TAIR database (https://www. arabidopsis.org/ (accessed on 24 January 2021)), the wheat genome data from the Ensemble database (http:/ / ensemblgenomes.org/, accessed on 24 January 2021), and the genome data of other species including quinoa were downloaded from the Phytozome plant genome database (https: / / phytozome.jgi.doe.gov/, accessed on 24 January 2021). The Uniport database (https://www.ebi.ac.uk/uniprot/, accessed on 24 January 2021) was used to query all the plant AUX/IAA proteins and obtain their protein sequence files. The AUX/IAA protein sequence was compared with the multispecies proteome data by BLAST method [16]. To cope with the potential issue of low sequence similarity of homologous sequences from distally related species, the hidden Markov model (HMM) profile of the AUX/IAA domain (PF02309) was downloaded from the Pfam database (https://pfam.xfam.org/, accessed on 24 January 2021). The HMM query in the HMMER v3.1 program was used to retrieve all AUX/IAAs from the 19 genomes with the threshold of an $e$-value $\leq 10^{-5}$. All results were submitted to the NCBI-BLAST tool (https:/ / blast.ncbi.nlm.nih.gov / Blast.cgi, accessed on 24 January 2021) for online retrieval to remove entries that did not belong to the family. The protein sequences of lower plants and higher plants' IAA were submitted to the orthovenn2 online tool [17] for orthologous identification. The maximum likelihood (ML) tree of multiple IAA proteins was conducted using the best-fitting in MEGA7, with Arabidopsis AUX/IAA sequences as outgroups. The optimal amino acid substitution model was deduced by the model selection (ML) program in MEGA: retain "JTT with Freqs (JTT+F)" for "Model/Method" and retain "Gamma distributed with sites $(\mathrm{G}+\mathrm{I})$ " for "Rates among sites". The default settings for other parameters were retained. The support values were estimated by 500 nonparametric bootstrap iterations. The IAA amino acid sequences of all species from different orthologous were searched in Pfam and CDD databases to find other known domains/motifs in addition to the AUX/IAA domain. In addition, MEME v4.9.0 [18] was used to identify the conserved motifs with the following parameters: width of a motif was 10-50 amino acids, and the number of motifs was 10. In addition, we identify key genes (YUC, TAA, TIR, ARF, PDC, $I D H, O G D C$, etc.) for quinoa auxin signal and tricarboxylic acid cycle pathway via BLASTp and online database annotation from quinoa genome annotation, combined with sequences of Arabidopsis related genes in the TAIR database. Details of these genes are recorded in Table S12.

\subsection{Chromosome Distribution, Gene Structure, and Syntenic Analysis in Quinoa and Other Plants}

Gff files and sequencing files were used to obtain chromosome localization and gene structure information of CqIAAs and to visualize data through TBtools [19]. To identify homologous blocks in different species, the syntenic analysis maps between the CqIAAs and AUX/IAAs from other plants were constructed with Dual Synteny Plotter software (https:/ / github.com/wyp1125/MCScanx, accessed on 24 January 2021) [20].

\subsection{Identification of Collinearity and Specific Duplication Events}

According to previous studies, gene collinearity analysis was performed using default parameters in MCScanX [21,22]. We used the Duplicate Gene Classifier program in MCScanX to speculate on different types of duplicated genes. We found potential anchoring points (E-value $<10^{-5}$; top 5 matches) between each possible pair of chromosomes in multiple genomes using BlastP. The loose E-value threshold accommodates a high differentiated 
evolution of duplicated genes resulting from polyploidization millions of years ago. Each species protein sequences are searched for their own or other species genome sequences. The homologous blocks in each genome and those between the genomes of different species were determined with Colinearscan (maximal gap $\leq 50$ genes; $p$-value $<0.05$ ) [23]. With reference to previous studies, the key parameters for the maximum gap between adjacent genes on chromosomes showing collinearity with genes on the corresponding chromosome sequence was set to 50 intervening genes [24].

\subsection{Gene Ontology (GO) and KEGG Pathway Annotation}

$\mathrm{GO}$ analysis was performed using the Blast2GO gene ontology analysis tools (http: / / www.blast2go.com, accessed on 24 January 2021) to determine the molecular function of all genes [25]. KEGG pathway enrichment in quinoa using KOBAS online tool 3.0 (http://kobas.cbi.pku.edu.cn/kobas3, accessed on 24 January 2021) [26].

\subsection{Trend Analysis of Gene Expression}

STEM v1.3.7 [27] were used to analyze the transcriptome data of different quinoa tissues based on the FPKM values under default parameters. a total of 49 expression pattern matrices were obtained, of which 14 were statistically significant trend modules. Detailed expression data and Profiles information are recorded in Table S8.

\subsection{Correlation Network Construction of CqIAAs and Other Metabolic Pathways}

Referring to previously reported methods, the interaction networks of CqIAAs with Gene of auxin biosynthesis, auxin signaling and TCA cycle pathways genes, auxin signaling, photosynthesis, and flavonoids under all different stresses were constructed by using the $\mathrm{R}$ package imsbInfer in GitHub. The associations were calculated using the Pearson correlation measure, and the red and blue edges represent positive and negative correlations.

\subsection{Calculation of the Synonymous and Nonsynonymous}

Using KaKs Calculator 2.0 to calculate the synonymous (Ks) and nonsynonymous (Ka) substitutions of each gene pair and their ratios (Ka/Ks) [28].

\subsection{Gene Expression Analysis in Two Kinds of Quinoa}

Publicly available C. quinoa RNA-Seq datasets were obtained from previous reports and the SRA (Sequence Read Archive, Shenzhen, China, https:/ / www.ncbi.nlm.nih.gov/ sra, accessed on 24 January 2021). These data were analyzed for the expression patterns of the identified AUX/IAAs and related metabolic pathway genes. Materials from fruit (SRS2464890), flower (SRS2464889), leaf (SRS4026093, SRS4026095), stem (SRS2464891), root [29], and materials with different fruit sizes [30] were used to examine the expression patterns and regulatory network. The expression level was normalized, and log2transformed was used for producing the heat map using the heatmap in TBtools. Using $\mathrm{R}$ packet limma (https:/ / github.com/wolski/imsbInfer, accessed on 24 January 2021) to analyze the differential expression of RNA-seq data, Foldchange $>2$ and $p$-value $<0.05$ were selected as the screening thresholds of significantly different genes. The heat maps and volcanic maps were also drawn by TBtools (v1.09854).

\subsection{Detection of Physiological Indexes after Stress Treatment}

The samples were divided into four groups: large-fruit quinoa control (LF + N), small fruit quinoa control $(S F+N)$, large-fruit quinoa nitrogen deficiency treatment $(L F-N)$, and small-fruit quinoa nitrogen deficiency treatment (SF - N). Each group had 15 plants, and 5 plants with the closest growth were randomly selected as biological repeats, and the average of 5 technical repeats was selected as the final result and recorded in Table S14.

The determination of the physiological parameters mainly refers to the method of Zhang et al. [31]; the specific determination method is as follows: Fresh leaves of $0.5 \mathrm{~g}$ 
were submerged into $90 \%$ acetone in an avoiding light environment for $24 \mathrm{~h}\left(4{ }^{\circ} \mathrm{C}\right)$. The absorbance of the solution was measured at 646.6 and $663.6 \mathrm{~nm}$ with a spectrophotometer (AOE Instruments, Shanghai, China), and then the calculations of chlorophyll content were made with reference to previous reports [31]. The amount of xylogen in the sample was determined by measuring the absorbance of reactants at $280 \mathrm{~nm}$ via perchloric acid oxidation method using a xylogen content assay kit AKSU010M (BOXBIO, Beijing, China); using a total pectin content assay kit AKSU069M (BOXBIO, Beijing, China); through the carbazole ratio method, the sample reacted with the carbazole reagent under concentrated sulfuric acid, and the absorbance of the generation was determined at $530 \mathrm{~nm}$ to determine the content of the pectin. The fresh leaf or root tissues of different treatments were homogenized with potassium phosphate (PBS) buffer $(\mathrm{pH}=7.4)$ under $0{ }^{\circ} \mathrm{C}$ precooling conditions. The homogenate was centrifuged at $20,000 \times \mathrm{g}$ for $20 \mathrm{~min}$ at $4{ }^{\circ} \mathrm{C}$, and the supernatant was collected for testing. Determination of the physiological parameters of plant stress was tested via the kit produced by Nanjing Jiancheng Institute of Bioengineering, taking malondialdehyde (MDA) content as assessment index of lipid peroxidation, and MDA content was determined by the thiobarbituric acid (TBA) method though recording the absorbance at 600,532 , and $450 \mathrm{~nm}$; superoxide dismutase (SOD) activity was determined by nitroblue tetrazolium; catalase (CAT) activity was determined by UV spectrometry method; peroxidase (POD) activity was determined by guaiacol method; hydrogen peroxide $\left(\mathrm{H}_{2} \mathrm{O}_{2}\right)$ content was quantitated by the hydroxylamine method; and the content of ascorbic acid (Vc) was determined by iron reduction/antioxidant and ascorbic acid (FRASC) method [31].

\subsection{Detection of Gene Expression after Stress Treatment}

We determined the expression level of C IIAA and related pathway genes (YUC, TAA, TIR, ARF, IAA, PDC, IDH, OGDC, and $M D H$ ) before and after nitrogen deficiency stress in two quinoa young roots by qRT-PCR. Primer 3 (http:/ / frodo.wi.mit.edu/, accessed on 24 January 2021) was used to design primers (Table S16). The elongation factor 1 alpha $(E F 1 \alpha)$ gene was used as an internal reference gene [32]. In our study, five seedlings with similar growth were selected from each treatment as a biological replicate. Total RNA of two quinoa (LF and SF) roots before and after nitrogen starvation was extracted using RNA Easy Fast DP452 kit (Tiangen, Beijing, China). The cDNA was synthesized with $1 \mathrm{mg}$ RNA samples using a PrimeScript RT reagent kit (TaKaRa, Dalian, China) with gDNA Eraser (TaKaRa) and SYBR Premix Ex Taq II (TaKaRa). The mRNA expression level in four samples $(\mathrm{LF}+\mathrm{N}, \mathrm{SF}+\mathrm{N}, \mathrm{LF}-\mathrm{N}$, and $\mathrm{SF}-\mathrm{N})$ was detected via qPCR experiments and obtained acquired gene expression information by the $2^{-\Delta \Delta C t}$ method [33]. During the experiment, each biological replicate was set up with three technical repeats. The mean value of the technical repeats was taken as the final result, and the three biological repeats were statistically tested.

\subsection{Statistics}

All data were analyzed using the Origin Pro 2020 statistics program, and the means were compared by the least significant difference test at the 0.05 level of significance. Error analysis is based on data from 3 biological replicates and 3 technical replicates.

\section{Results}

3.1. AUX/IAA Family Originated in the Early Stage of Plant Landification and with Conservative Evolutionary Pattern in Higher Plants

Integrating hormone signaling from different plants during landification is complicated because of intense environmental changes. This longstanding problem is even more extreme in terrestrial plants because of their often complex and polyploid genomes as well as past whole-genome duplication events [34]. To further enhance our understanding of the evolution of hormone signals during the process of plant landification, a total of 495 auxin-reactive proteins (AUX/IAA) were identified from 19 plants of agricultural 
and evolutionary importance. AUX/IAA proteins were not found in two aquatic plants (Volvox carteri and Chlamydomonas reinhardtii) (Figure 1A,B, Table S1), and this suggests that AUX/IAA protein family may have originated after plants have colonized the land. We also predicted the physical properties of the identified AUX/IAA proteins, and the molecular weights and isoelectric points of different AUX/IAA proteins were different (Figure 1A, Table S1). Further, we searched for direct homologous proteins from AUX/IAA proteins of lower and higher plants, respectively. According to the conserved ancestral copies shared among different plants, the AUX/IAA proteins in lower plants were divided into nine orthogroups (Figure 1B). The lower plant orthogroup 01 (LG01) covered all plant AUX/IAA proteins, while in the other eight LGs with some plant AUX/IAA proteins were lost. The integration of Arabidopsis AUX/IAAs (AtIAAs) into a homologous search of AUX/IAAs in lower plants is intended to better understand the transition evolution of IAA proteins in plants. Notably, $15(60 \%)$ Arabidopsis AUX/IAA proteins are in LG01, suggesting that AtIAAs share ancestral genes with early lower plants. The molecular evolution tree based on the sequence alignment of AUX/IAA proteins shows that the LG01 has a more complex topology across multiple lower plants. The structural analysis of protein motifs in orthogroups reveals molecular evolution patterns of low plant IAA family differences. IAA proteins in most lower plants have highly conserved motifs $1,2,3$, and 5, while we did not find motifs 3 and 5 in some members (85038 and 36236) of the Selaginella moellendorffii. An interesting phenomenon is that complete motifs 1-10 only exist in all Physcomitrella patens family members. However, these unique motifs are once again lost in Arabidopsis IAA proteins (Figure 1C). Moreover, the GO enrichment results for all identified proteins in the LG01 shared by lower plants showed that the proteins in the LG01 were involved in the biological process of the response to auxin, which further verified the reliability of the family identification results (Figure 1D,E). The above conclusions reveal the early origin of conserved structures of IAA proteins. The evolution of new motif structures in Physcomitrella patens suggests the structural diversity and functional explosion of IAA proteins in bryophytes. However, as plants evolved, these nonshared motifs were lost in higher plants represented by Arabidopsis (Figure 1B,C).

Furthermore, we used the same method to systematically classify the IAA proteins of 12 higher plants (Figure 1F). There are more IAA family members in domesticated crops such as G. max, O. sativa, Triticum aestivum, Zea mays, and C. quinoa; notably, up to 16 G. max IAA proteins were classified into higher plant orthogroups 01 (LG01), and fewer AUX/IAAs in early differentiated species such as coffee and grapes. (Figure 1F). Only HG04 conforms to the characteristics that cover all higher plants' AUX/IAAs, in sharp contrast to most other HGs. IAA proteins in most species contain conserved motifs 1-10 except for all Fagopyrum tataricum IAA proteins in the HG01 that lost motif 9, one maize IAA protein which lost motif 6, and one quinoa IAA protein which lost motif 5 (Figure 1G). The IAA protein structure in other HGs is similar to that in HG01, except for the minority protein with nonconservative motifs' loss, the IAA proteins in most species have similar structural composition, suggesting that the evolutionary pattern of IAA proteins in higher plants is conserved (Figure 1G-K). 
A
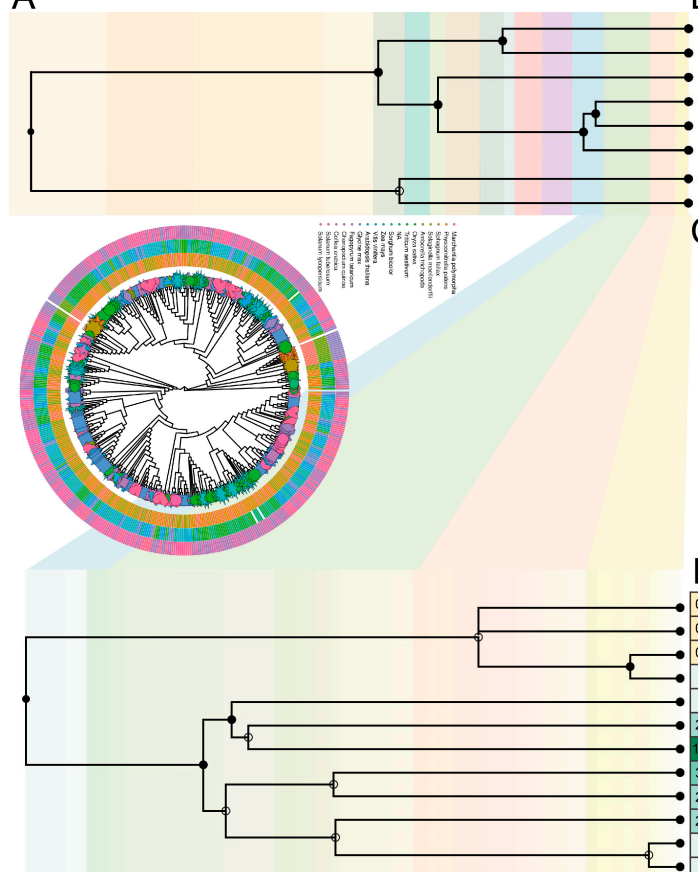

$\mathrm{F}$

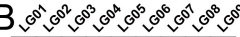

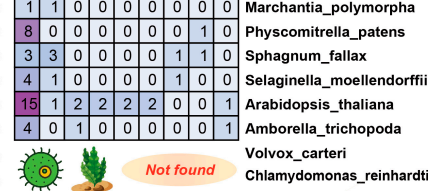

C

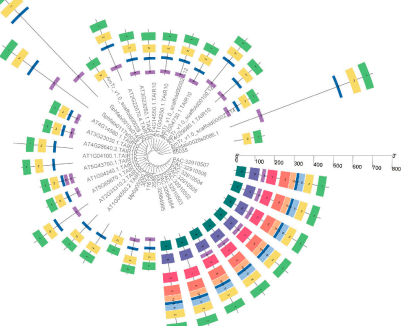

D

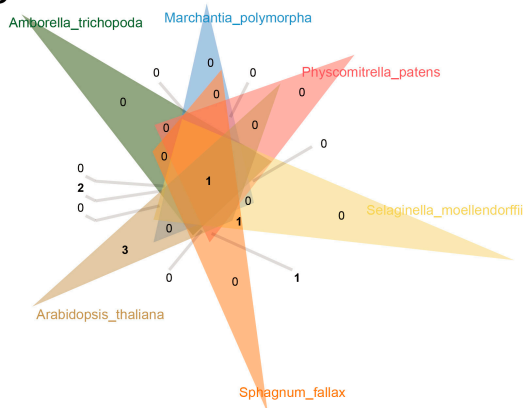

E

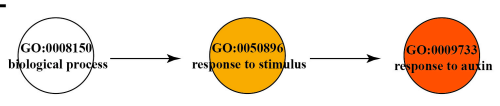

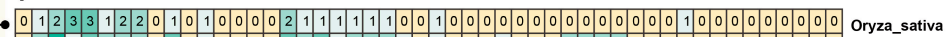

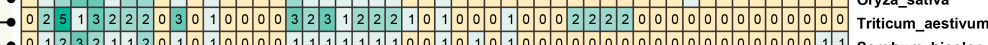

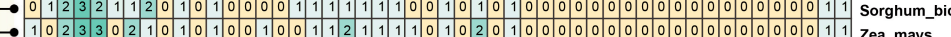

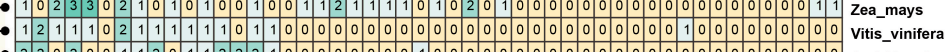

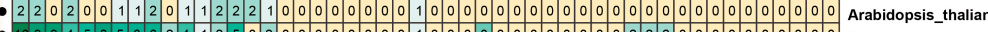

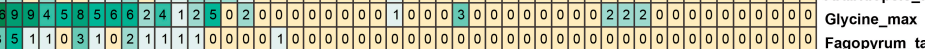

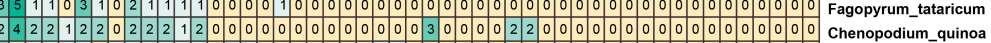

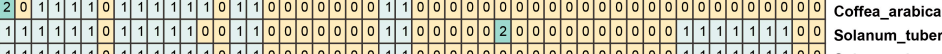

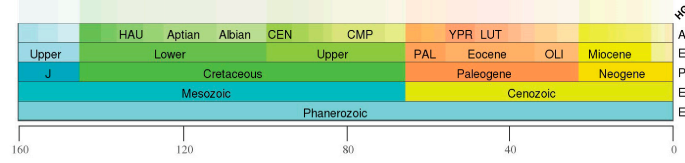

G

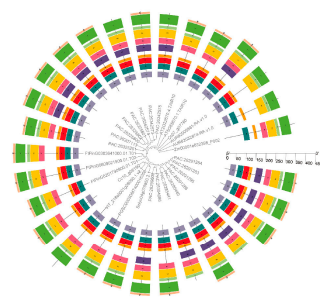

$\mathrm{H}$

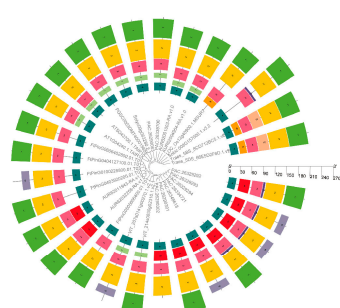

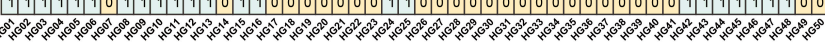
olanum_tuberosum Ages
Epochs

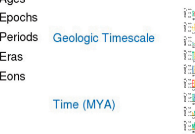

I
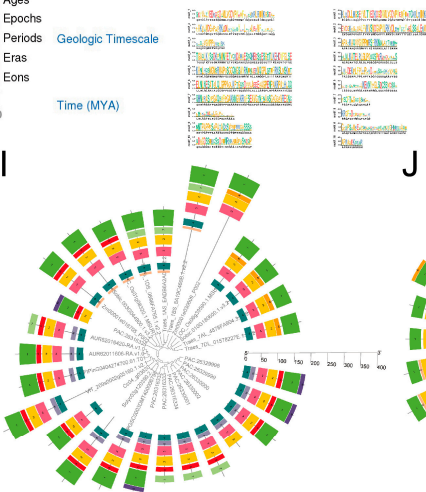
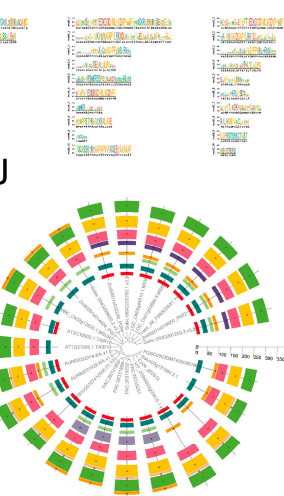

$\mathrm{K}$
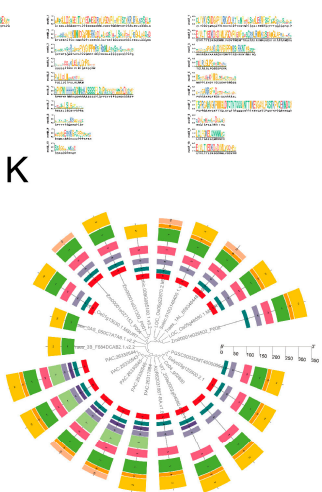

Figure 1. Phylogeny, diversity, and motif compositions of AUX/IAA proteins in 19 species. (A). Phylogenetic tree of 495 AUX/IAA from 19 species and their amino acid length, isoelectric point, and molecular weight. Different color blocks at the end represent the species to which the protein belongs, and the internal and external heat maps represent the amino acid length, isoelectric point, and molecular weight, respectively. The specific data are listed in the Table S1; (B). AUX/IAA protein orthogroups of lower plants and Arabidopsis; (C). Phylogenetic tree and motif structure in orthogroups group 01 (LG01) shared by lower plants; (D,E). Sharing of orthogroups among lower plants and GO enrichment analysis of all proteins in LG01; (F). AUX/IAA protein orthogroups of higher plants; (G-K). Phylogenetic tree and motif structure of AUX/IAA protein in HG01-HG05 middle and higher plants.

3.2. Structural and Evolutionary Analysis of the CqIAA Family of Halophytes Crops Quinoa Reveals the Family's Generally Conserved Evolutionary Model and Rapidly Evolving Genes Selected by a Strong Environment

Quinoa has experienced long domestication and complex hybridization events in history and is a good material to study the adaptation mechanism of the agroecological environment after crop domestication [35]. To investigate the molecular evolution of the IAA protein family within the quinoa genome, we constructed phylogenetic trees in 37 CqIAAs and found that CqIAAs evolved into complex topology (Figure 2A). The promoter region sequences of $2000 \mathrm{bp}$ upstream of each IAA were further extracted for predicting potential hormone and environmental response elements (Figure 2B, Table S3). Among the IAA families, light response elements are the most, followed by stress response elements, 
hormone response elements, and other environmental response elements (Figure 2B). Further, we performed gene structure and protein motif analysis for all $C q I A A s$ (Figure 2C,D). The number of introns in the CqIAAs ranges from 1 to 5, and the CDS region of each gene contains at least one family-specific conserved structure annotated as AUX/IAA. CqIAA proteins with close phylogenetic relationships have similar motif composition, although some protein motifs are developed or lost in some CqIAAs. For example, motif 9 (purple box) exists only in AUR62008800 and AUR62025439, and motif 7 (red box) is detected only in AUR62002810 and AUR62014118 (Figure 2D). Moreover, motif 2 (yellow box) is present in almost all CqIAA proteins and is their conserved motif. Interestingly, motif 2 in AUR62043556, AUR62016513, AUR62005398, and AUR62004953 are missing (Figure 2D). The gain and loss of the motif may indicate the evolution of the function, which needs further discussion.

A

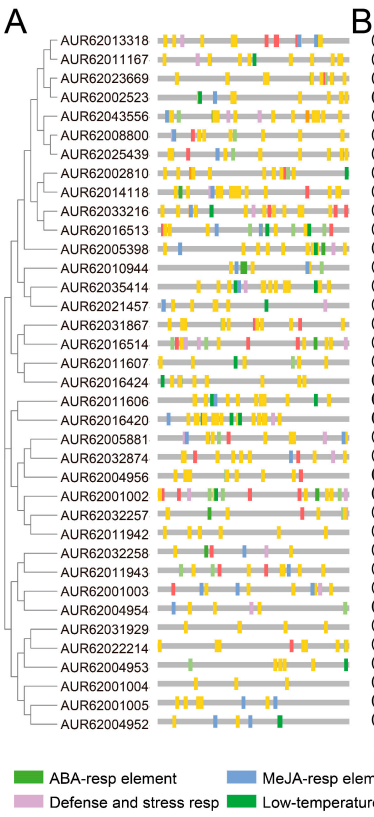

E

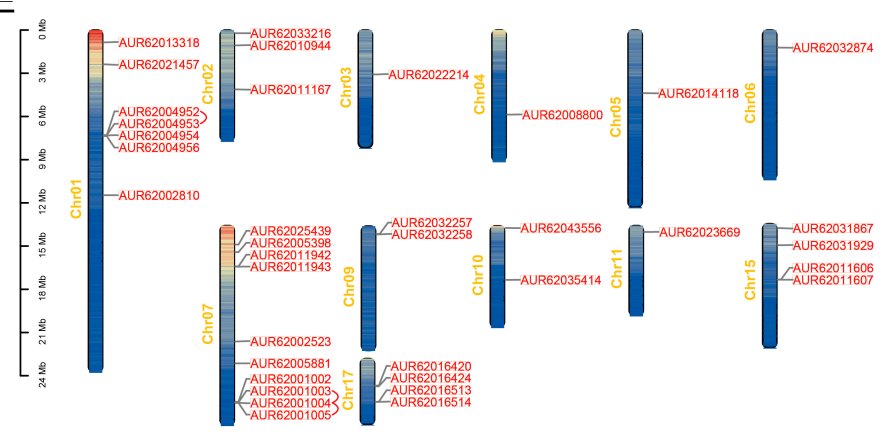

B
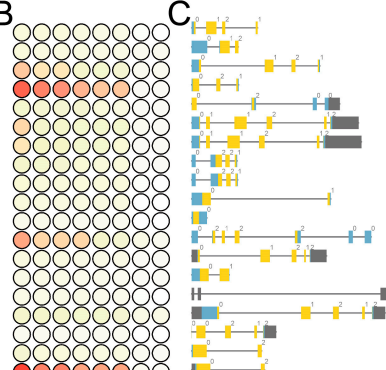

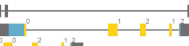
Auxin-resp element
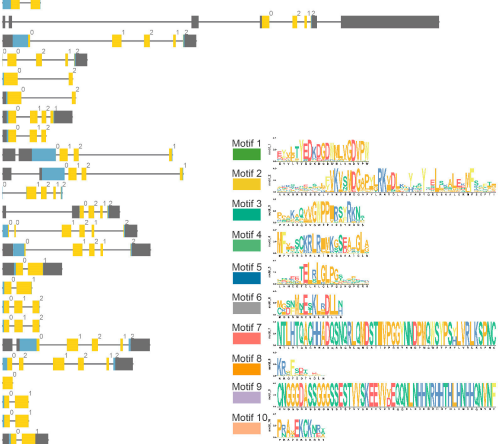

$4000 \quad 6000 \quad 8000$ Drought-inducibility elemen CDS UTR IUXIAA

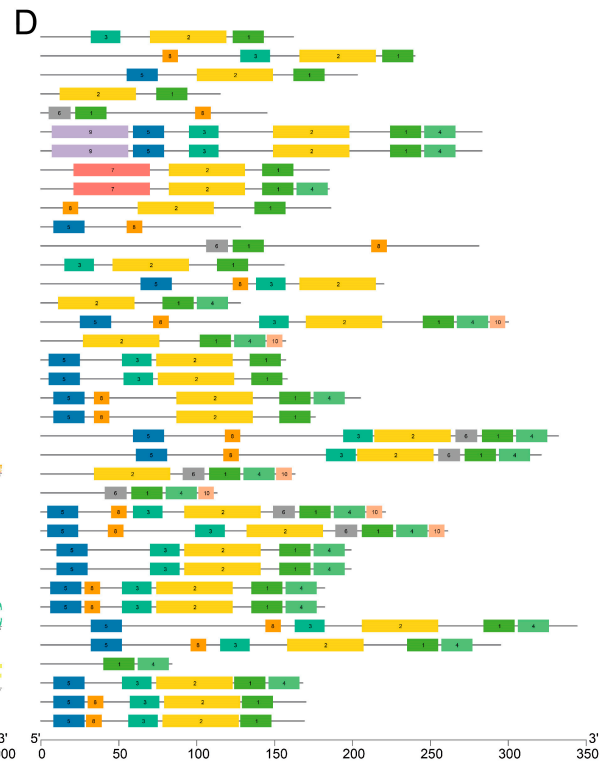

F

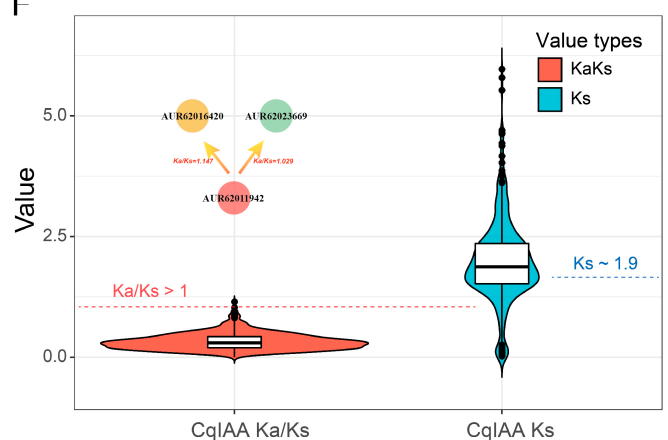

Figure 2. Phylogeny, gene structure, protein motif, chromosomal localization, and evolutionary analysis of quinoa CqIAA protein family. (A). Cis-acting element types and distributions in 37 CqIAAs promoter regions. Different color blocks represent different types of cis-acting elements and are distributed within the $2000 \mathrm{bp}$ promoter region; (B). Classification statistics of different cis-acting elements; (C). The gene structure of 37 CqIAAs is shown: blue, gray, and yellow box represent coding region, noncoding region, and AUX/IAA domain, respectively; black lines represent introns; (D). CqIAAs' motif structure, the boxes of different colors represent 10 conserved motifs; (E). CqIAAs' chromosome mapping, the red arcs represent tandem duplication gene pairs; (F). Ks and Ka/Ks rate between CqIAA pairs, two pairs of Ka/Ks > 1 IAA gene pairs are shown above the red dotted line; the blue dotted line indicates that the peak Ks distribution of the CqIAA pairs is close to 1.9. 
Chromosome mapping through Gff file revealed that 37 CqIAAs were unevenly distributed on 12 of 18 chromosomes, and 3 pairs of tandem duplication gene pairs were detected (Figure 2E) which may suggest that replication events play a key role in the family's expansion. The synonymous (Ks) and non-synonymous (Ka) substitutions of all CqIAA sequences were further calculated. The results showed that the vast majority of CqIAAs were subjected to environmental purification selection, but the $\mathrm{Ka} / \mathrm{Ks}$ of two pairs of genes (AUR62011942-AUR62016420 and AUR62011942-AUR62023669) was greater than 1 (Figure 2F, Table S4). Interestingly, most gene pairs with $\mathrm{Ka} / \mathrm{Ks}$ value close to 1 are AUR62011942 involved in (Table S4), which suggests that the gene may have important functions related to growth and development regulation and evolve rapidly under environmental selection. We also calculated the synonymous substitution (Ks) rate between CqIAAs, in preparation for a subsequent estimate of the expansion time of this family (Figure 2F, Table S4).

\subsection{Evolutionary Analysis of IAA Proteins in Typical Polyploid Agricultural Crops Emphasizes the Contribution of Polyploidy to AUX/IAA Family Expansion}

The expansion of the gene family, often driven by gene duplication of different ways, has repeatedly played irreplaceable roles in providing the raw material for the adaptive evolution of plants in changing complex environments [36]. Syntenic analysis between chromosomes helps us to understand the duplication of gene clusters within segments caused by ancient polyploidy events. We selected a heterotetraploid crop quinoa, a hexaploid grain T. aestivum, and hybrid polyploid Brassica napus as the materials for genomic collinearity analysis (Figure 2A-C, Table S5). The results showed that 15, 94, and 141 syntenic AUX/IAA gene pairs were found in the three plant genomes, respectively (Figure 2A-C, Table S5). Interestingly, the syntenic pairs in the three subgenomes of hexaploid T. aestivum had a 2:1 ratio, which is consistent with the previously reported characteristics of the wheat MADS family [37], confirming that the AUX/IAA family is preserved with the fusion of different subgenomes of wheat (Figure 2B). Although quinoa and B. napus are also allopolyploid with different subgenomes, the synteny and evolutionary retention patterns of AUX/IAA family are significantly different than that of T. aestivum.

In addition to synteny gene pairs within species, synteny homologs between species are widespread and often functionally related [38]. We analyzed the syntenic AUX/IAA pairs of quinoa with other higher plants, especially with gramineous plants (Figure 2D, Table S6). Visualization showed the total syntenic relationships between quinoa and $O$. sativa (two pairs), T. aestivum (only one pair), S. bicolor (two pairs), Z. mays (two pairs), Arabidopsis (five pairs), and F. tataricum (seven pairs). Moreover, this suggests that species with closer phylogeny have more collinearity IAA pairs, although the syntenic relationship of AUX/IAAs are not strong among multiple species, which suggests that segment duplication is not the main cause of IAA family's expansion in the plant kingdom (Figure 2D, Table S6).

Early studies have confirmed that another important driver of plant genome evolution is whole-genome duplication (WGD) [39]. We speculated that the expansion of the IAA family after plant landification may be due to WGD events. The Ks values of homologous pairs in syntenic regions of the genome, as well as the mean Ks values of individual syntenic blocks, indicated that WGD events had occurred in the evolutionary history of representative species (Figure 2E). The Ks density plot of representative species showed that the quinoa genome experienced a late WGD event, but slightly earlier than the WGD event shared by angiosperms, this may be closely related to a hybridization during quinoa domestication, which may be closely related to a hybridization during quinoa domestication [15]. Interestingly, the Ks peak of quinoa genome is close to the Ks peak of CqIAAs (Figure 2F), which may suggest that the IAA family expands with the doubling of the quinoa genome. To further investigate whether polyploidy events promote the expansion of the IAA family in representative species, we used MCScanX to identify repeat types of AUX/IAAs in eight plants, including quinoa (Figure 2F, Table S7). As a result, most of the AUX/IAAs in C. quinoa, B. napus, Arabidopsis, O. sativa, and Sorghum 
bicolor were associated with polyploidy (Figure 2F, Table S7). The combined results show that the expansion of AUX/IAA family in higher plants represented by quinoa is mainly due to polyploidization events, although AUX/IAAs in some species (T. aestivum and Z. mays) have other expansion patterns (dispersed and tandem duplication).

\subsection{RNA-Seq Data Analysis of Quinoa Root Provides a Reference for the Network of Hormone Signals and Environmental Regulation Formed by AUX/IAAs}

The family of polyploidy reserved transcription factors often contributes to the adaptive evolution of plants to complex environments [40], and the perception and response of these environmental factors are more coupled through complex root signaling networks $[40,41]$. We collected RNA-seq data sets from different tissues of quinoa from the NABI GEO database for expression trend analysis, and the normalized expression data were used to perform the clustering (Figure 3A, Table S8). The results showed that 14 statistically significant model profiles (colored profiles) were identified in 49 profiles generated (Figure 3A). Of these 14 profiles, three special model profiles (profiles 41, 42, and 44) were identified with significantly higher expression levels in roots than in other tissues (Figure 3B-D). KEGG pathway enrichment analysis of genes in profiles 41,42 , and 44 via KOBAS online database (Figure $4 \mathrm{~F}-\mathrm{H}$, Table S8) was conducted. Phenylpropanoid biosynthesis (ko00940), ubiquinone and other terpenoid-quinone biosynthesis (ko00130), plant hormone signal transduction (ko04075), and other entries were significantly enriched in profile 41 (Figure $4 \mathrm{~F}$, Table S8); the enrichment results of amino sugar and nucleotide sugar metabolism (ko00520), plant hormone signal transformation (ko04075), ubiquitinmediated proteolysis (ko04120), and biosynthesis of secondary metabolites (ko01110) in profile 42 have attracted our attention (Figure 4G); ubiquitin mediated proteolysis (ko04120), plant hormone signal transformation (ko04075, Table S8), amino sugar, and negative sugar metabolism (ko00520) in the profile 42 also have a significant rich factor (Figure $4 \mathrm{H}$ ). IAA is closely related to ARFs' protein-protein interaction and ubiquitination-mediated protein degradation pathway in the auxin signaling pathway, and auxin signal is also an important component of plant hormone signal transduction [5,42,43], which further leads to our strong attention to root auxin signaling process.

Moreover, a coexpression network analysis of root-specific expression genes and 37 IAA genes showed that 437 edges with significant correlation $(p<0.01)$ were generated when a total of 1019 nodes were input (Figure 4I, Table S9). When using the expression data of the other four organizations for correlation analysis, there are only 347 significant correlation edges $(p<0.01)$ generated after the same number of nodes input (Figure $4 \mathrm{I}$, Table S9). These findings confirm that the IAA family has formed a closer regulatory network in the root system, which is in contrast to the absence of such a correlation in expression data for four other organizations. Gene ontology (GO) analysis helps us to explore the potential functions of genes that are significantly related to IAAs, and we show the top 15 terms in three parts including biological processes (BP), cellular components (CC), and molecular functions (MF). The significantly enriched terms including protein modification process (GO:0036211) (BP terms) cell wall (GO:0005618), plant-type cell wall (GO:0009505) (CC terms) protein binding (GO:0005515), and hydrolase activity (GO:0016787) (MF terms), which have caught our attention because they again remind the potential role of auxin signaling in root growth and development (Figure 4J, Table S10). 

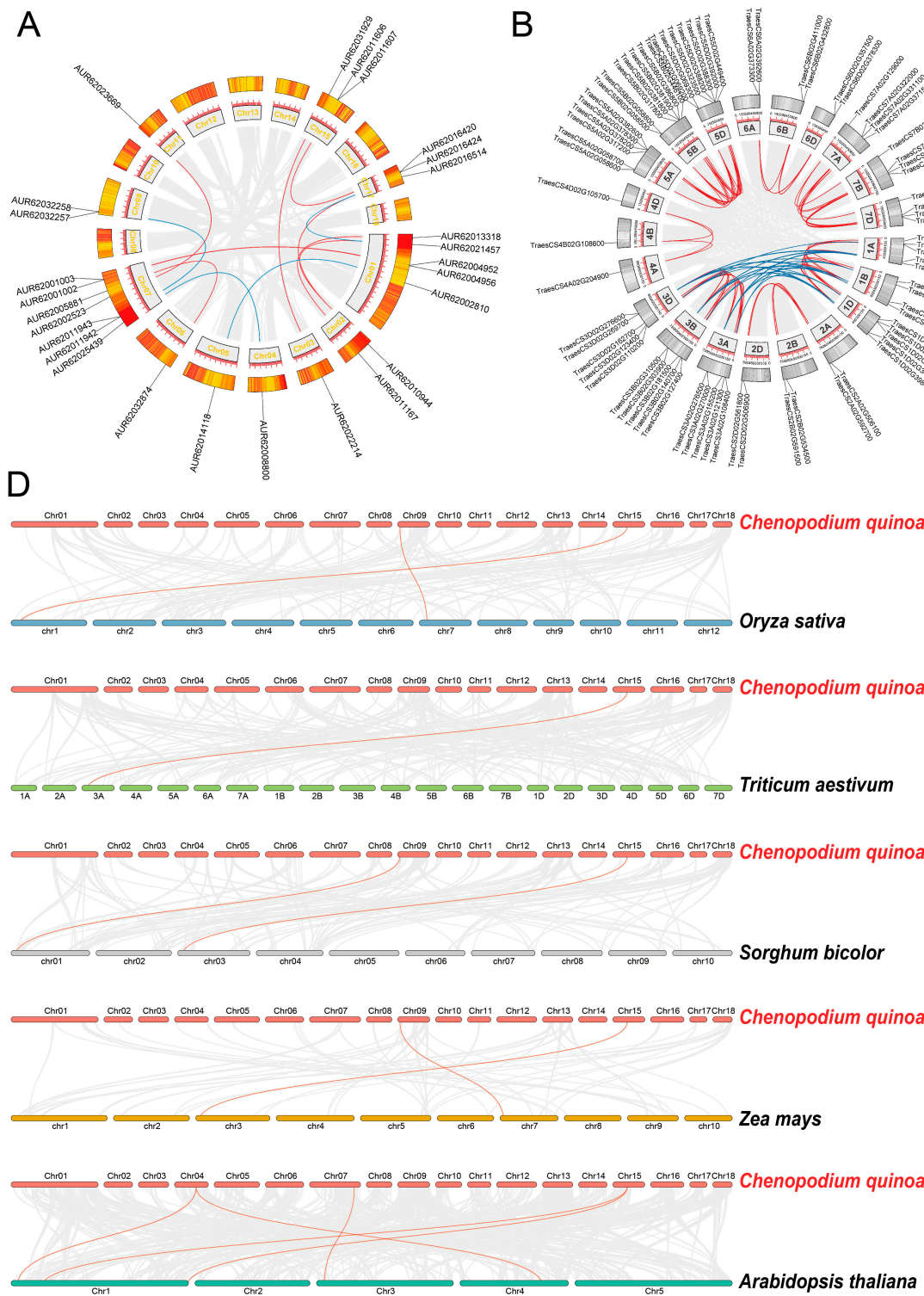

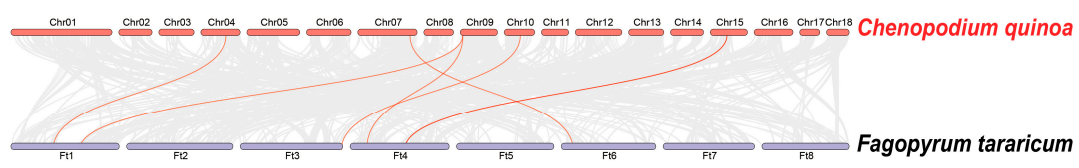

B

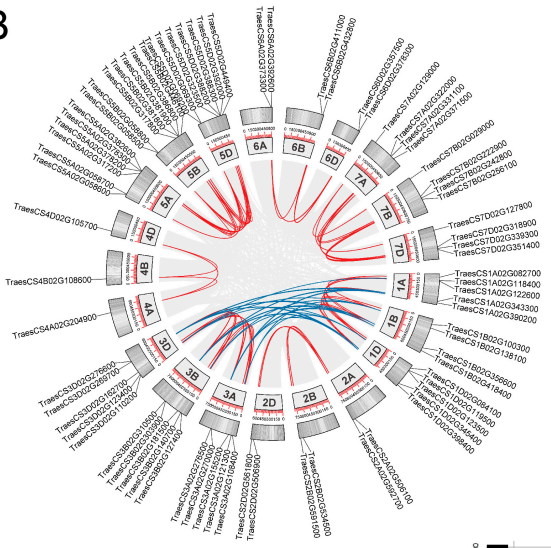

C

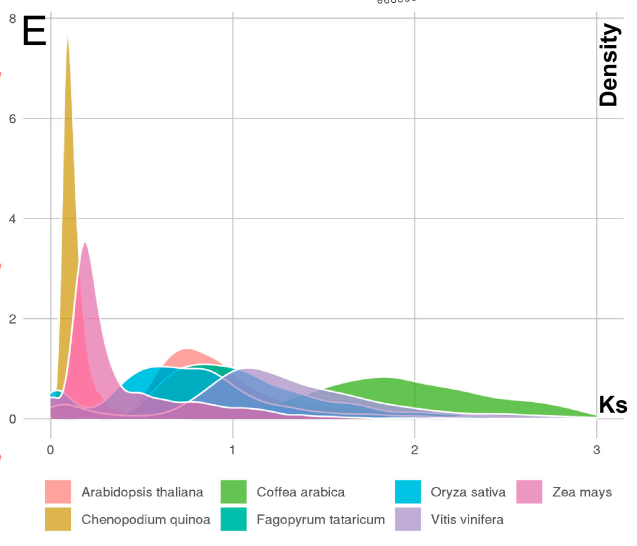

$\mathrm{F}$

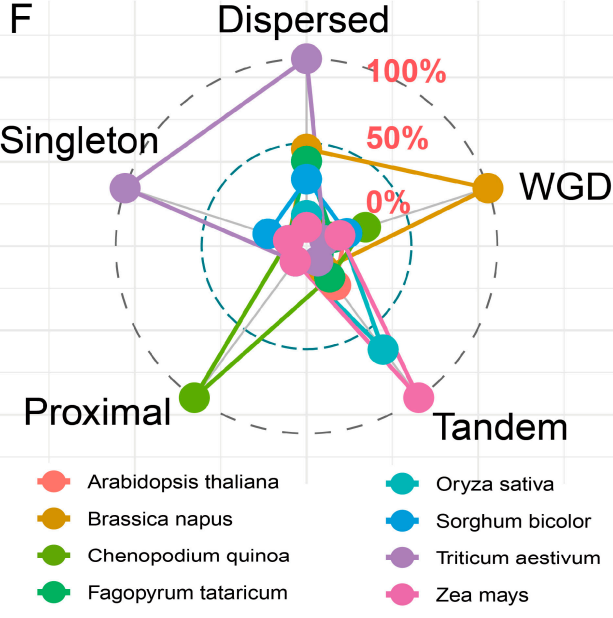

Figure 3. Schematic representations of the interchromosomal relationships of AUX/IAAs, gene duplication types of AUX/IAAs, and WGD events analyses in different species. (A-C). Synteny AUX/IAA pairs within the genome of quinoa, wheat, and Brassica napus. The red lines indicate collinear gene pairs from the same subgenome in polyploid species, and the blue lines indicate collinear gene pairs from different subgenome; (D). Synteny AUX/IAA pairs between quinoa and other 6 angiospermic genomes; (E). The proportion of gene pairs in each plant binned according to Ks values. A high proportion of orthologous gene pairs in plants showed similar rates of synonymous substitutions per synonymous site (Ks), indicative of a whole-genome duplication event; (F). Distribution of duplication types of AUX/IAAs in eight multispecies. Multiple collinearity scan toolkit (MCScanX) was used to detect duplication types to identify GRASs associated with ancestral polyploidization events (WGD or segmental). The AUX/IAAs related to polyploidy were inferred according to the collinearity persistence of the region around the selected genes. AUX/IAAs were divided into five groups, including dispersed, proximal, tandem, singleton, and WGD or segmental. 


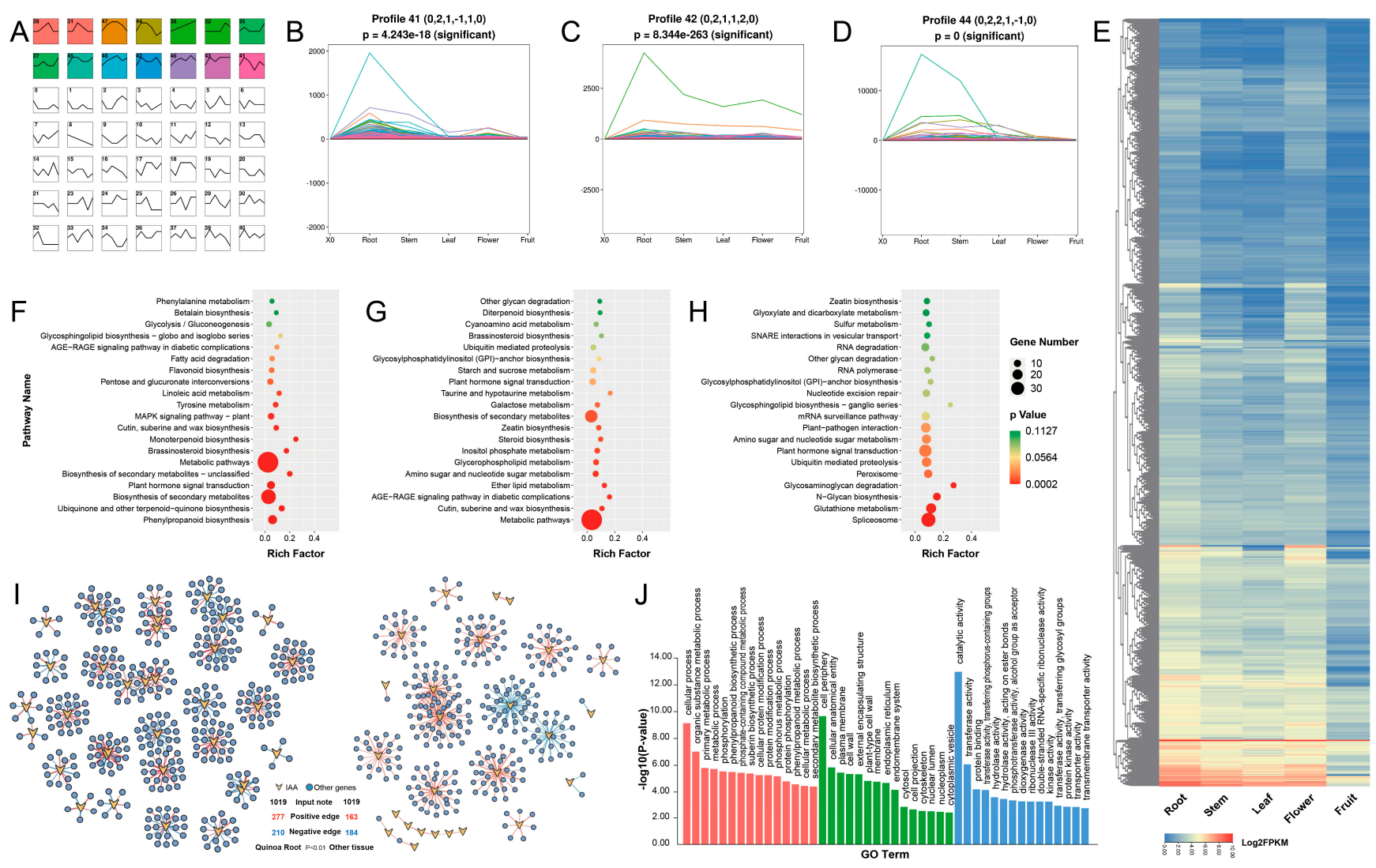

Figure 4. Search for highly expressed genes in quinoa roots, construction of networks associated and functional prediction of CqIAAs potential target genes. (A). The 49 expression trend maps based on the RNA-seq data of quinoa roots, the first 14 of which were labeled color profiles were statistically significant; (B-D). Display of expression trends of three highly expressed gene profiles (profile 41, 42 and 44) in roots; detailed data are recorded in the Table S8; (E). Heat maps showing in five tissues further confirmed that the transcription level of these genes in roots was significantly higher than that in other tissues; (F-H). KEGG pathway enrichment for three highly expressed gene profiles (profile 41, 42, and 44) in roots; Detailed data are recorded in the Table S8; (I). Correlation network between genes and CqIAAs formation in 3 profiles. The yellow diamonds represents CqIAAs, and blue circle represents the genes in 3 profiles. The red line represents positive correlation; the blue line represents negative correlation. correlation network is obtained by $p<0.01$ as threshold screening. Networks on the left and right are constructed using the RNA-seq data root and other organizations, respectively; detailed data are recorded in the Table S9; (J). GO enrichment of CqIAAs' target genes in correlation networks. red, green, and blue column distributions represent three terms. of biological processes, cellular components, and molecular functions. The top 15 entries in each term were chosen for presentation. Detailed data are recorded in the Table S10.

3.5. Among Quinoa with Different Fruit Phenotypes, AUX/IAA Family Formed a Robust Network of Correlations with Hormone Signaling and Energy Metabolism Pathways

Transcriptome changes of seed continue to affect the development of radicles and the interaction with the environment after germination [44]. For mining genes with potential regulatory functions for root-environment interaction, we first analyzed the RNA-seq data of quinoa seeds to understand the reserve resources available at the seed stage. We selected two quinoa seeds with significant differences $(p<0.001)$ in size and color, named large fruit (LF) and small-fruit (SF) quinoa according to their size (Figure 5A, Table S11), and we hierarchically clustered 37 CqIAAs expressed in two quinoa fruits based on the expression level (Figure 5B, Table S11). Eight IAA genes (AUR62005881, AUR62031867, AUR62004956, AUR62032874, AUR62025439, AUR62011942, AUR62008800, and AUR62001002) have high transcription levels in both large and SF quinoa, while six genes (AUR62035414, AUR62011167, AUR62016513, AUR62021457, AUR62023669, and AUR62002523) were not expressed in either fruit. Notably, AUR62011942 had significantly 
different expression patterns (Foldchange $>2, p<0.05$ ) in the two quinoa fruits (Figure 5B, Table S11), which may be a potential regulator of the differential phenotype between the two quinoa species.
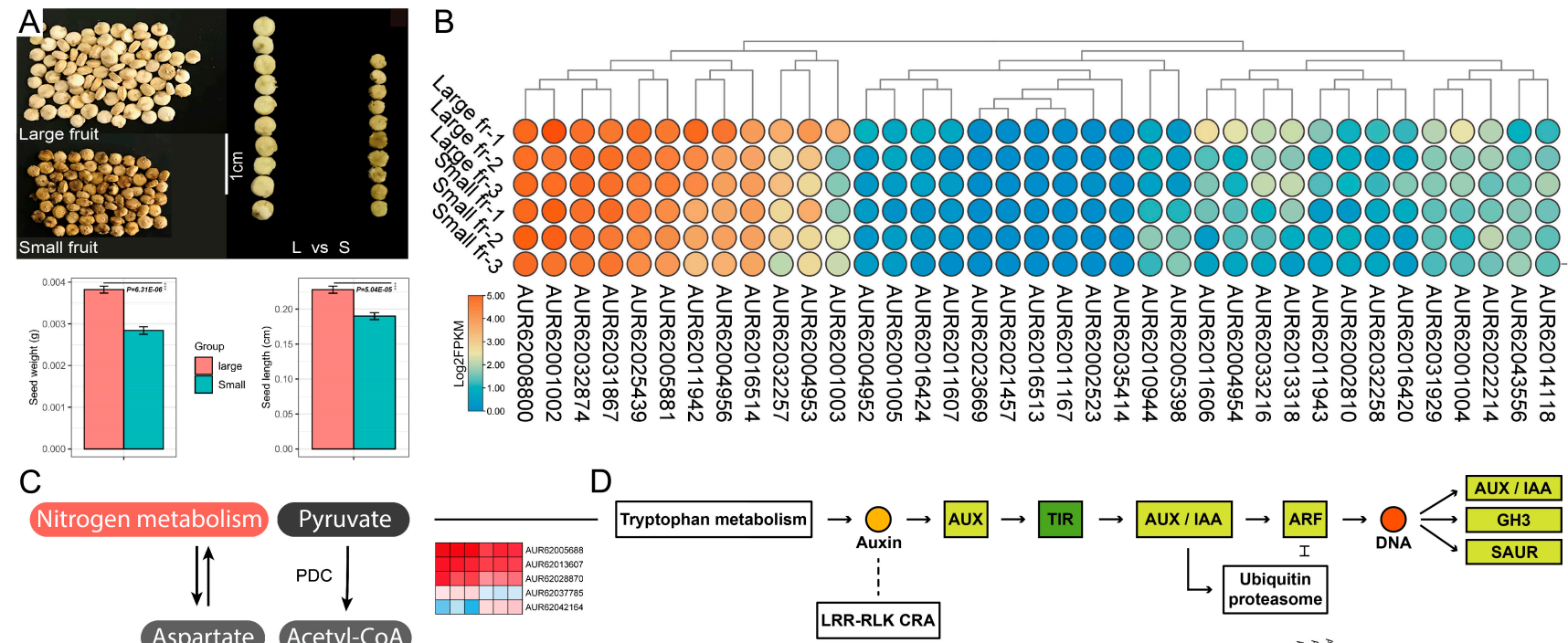

C
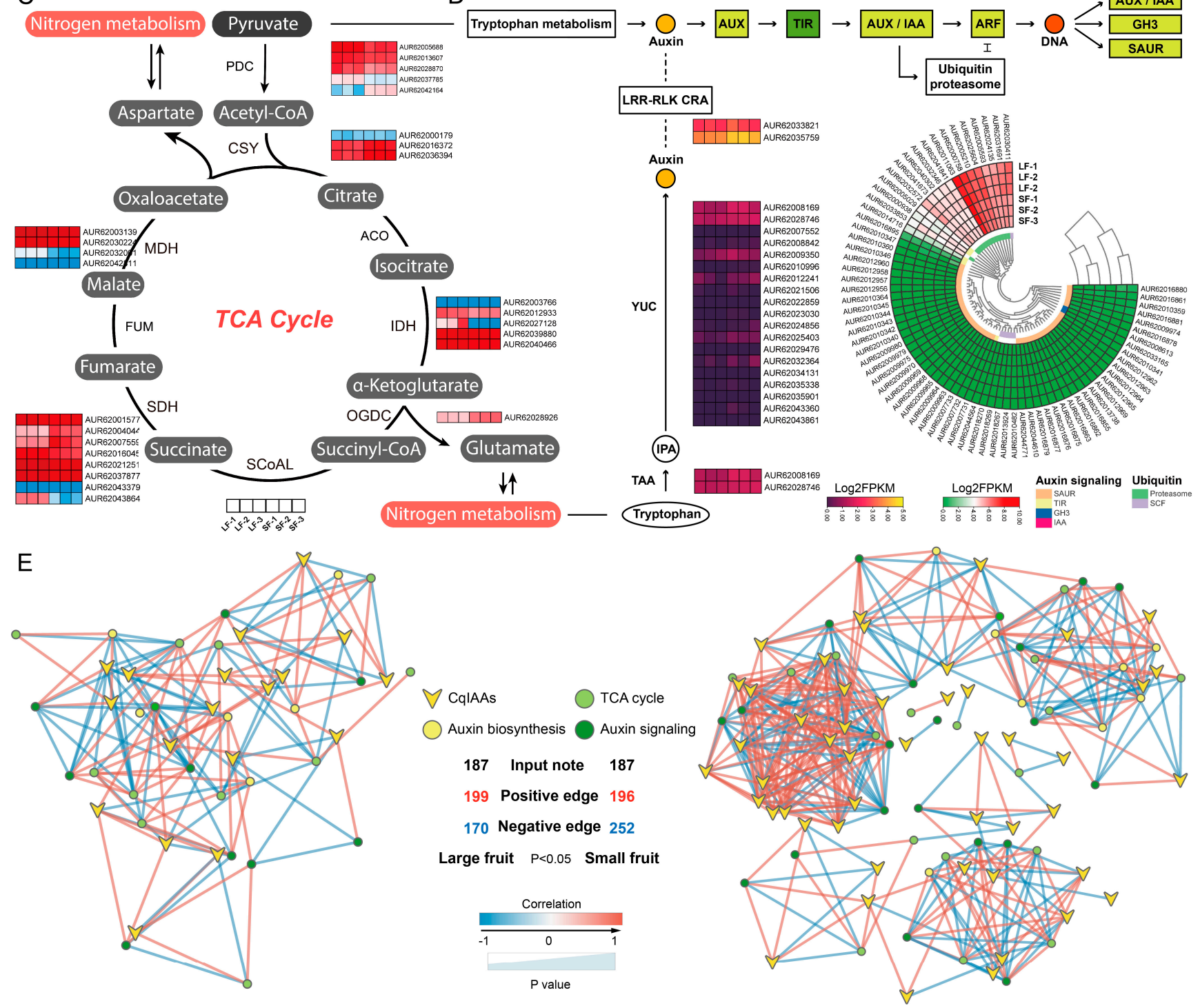

Figure 5. Morphological display, differential expression of CqIAAs, differential expression of auxin signaling and energy metabolism, differential expression of auxin signaling and energy metabolism pathway and sexual network with CqIAAs. 
(A). Morphological presentation and data comparison of quinoa with different seed sizes and colors. (B). Expression profiles of 37 CqIAAs in two quinoa seeds. According to the expression quantity (FPKM), the heat map is clustered (expression data are recorded in Table S11); (C). Expression profiles of TCA cycle pathway genes in two quinoa seeds (expression data are recorded in Table S12); (D). Expression profiles of auxin biosynthesis and signal transduction pathway genes in two quinoa seeds (expression data are recorded in Table S12); (E). Correlation network between CqIAAs and different pathway genes in two quinoa seeds (correlation data are recorded in Table S13). Yellow diamonds represent CqIAAs; different color circles represent auxin signaling and energy metabolism pathways. The red line represents positive correlation; the blue line represents negative correlation. Correlation network is obtained by $p<0.05$ as threshold screening. Networks on the left and right are constructed using RNA-seq data the small-fruit and LF quinoa seeds, respectively.

Energy and hormone metabolisms are the most vigorous biological processes in plant seed germination and seedling growth and development $[45,46]$. As a result, we further retrieved the gene expression data of the tricarboxylic acid cycle, auxin biosynthesis, and auxin signaling pathway from the transcriptome data sets of two quinoa fruits (Figure 5C,D, Table S12) to mine target genes that may be closely related to the IAA family. In the TCA cycle, pyruvate dehydrogenase (PDC) gene AUR62037785 upregulated in LF quinoa while AUR62042164 downregulated. Citrate synthase (CSY) is the rate-limiting enzyme in this pathway, but there is no significant difference in its expression in the two quinoa fruits. Furthermore, the expression level of isocitrate dehydrogenase (IDH) gene AUR62027128, succinate dehydrogenase $(S D H)$ gene $A U R 62043864$, and malic dehydrogenase $(M D H)$ gene AUR62032001 in LF quinoa were all higher than SF quinoa. Notably, the $\alpha$ ketoglutarate dehydrogenase (OGDC) gene AUR62028926 coupled carbon metabolism with nitrogen metabolism [47], and it had a higher expression level in SF quinoa than in LF quinoa, suggesting that SF quinoa may have potential nitrogen reserves in the early stages of germination (Figure 5C, Table S12). Unexpectedly, the expression levels of two key enzyme genes (TAA and YUC) of the auxin biosynthesis pathway in both large and SF quinoa were low (Figure 5D, Table S12). A recent report confirmed that LRR-RLK was able to respond to soil nitrogen signals and, coupled with auxin signals [48], had higher expression levels in both fruits. Moreover, the GH3, SAUR from the auxin signaling pathway were almost nonexpressed in both fruits, and the proteasome genes expression in the ubiquitination pathway is active, which is in contrast to that of the coding genes of the SCF complex which appear to be silent (Figure 5D, Table S12). Moreover, using transcriptome data from two quinoa seeds, we constructed a correlation network of genes CqIAAs, auxin biosynthesis, auxin signal transduction, and TCA cycle pathway genes (Figure 5E, Table S13). Interestingly, CqIAAs form a robust regulatory network with metabolic pathway genes. Hormone signaling and energy metabolism regulation network are more robust in SF quinoa, which suggests that it may have greater adaptability to a complex agroecological environment during germination (Figure 5E, Table S13).

3.6. Metabolic Regulatory Networks Formed by IAA May Help Induce Stress Responses of Root Systems of Quinoa Seedlings in Nitrogen Starvation Agroecological Environment

TCA cycle is the core pathway of cell energy metabolism, which also provides essential precursors for amino acid biosynthesis and maintains metabolic balance with carbon [49]. In order to verify whether the two quinoa seeds with different energy and hormone signal networks formed different environmental responsiveness after germination, we induced the germination of the two quinoa seeds under the same conditions. Quinoa seedlings were treated with water culture under nitrogen-deficient environment when the cotyledons were fully expanded. The results showed that after two weeks of a nitrogen-deficient culture, the roots of both quinoa seedlings were elongated (Figure 6A, Table S14). Specifically, the root elongation of SF quinoa before and after stress is significantly different, which is in sharp contrast to LF quinoa; there was no significant difference in the increase in root biomass between the two species (Figure 6B, Table S14). The determination of cell wall components in the root system of two kinds of quinoa showed that the decrease in pectin in SF quinoa root was more obvious than that in the LF quinoa root system; the increase in xylogen in 
the root of LF quinoa resveratrol was more obvious than that of the SF quinoa (Figure 6C, Table S14). We also determined the chlorophyll content in the leaves of quinoa. The results showed that the chlorophyll A, chlorophyll B, and total chlorophyll content in the leaves of the two kinds of quinoa decreased after stress, and the decrease in LF quinoa was more significant (Figure 6D, Table S14). Through the principal component analysis (PCA) of the compound content of two kinds of quinoa under two treatments, it was found that the above indexes of the two kinds of quinoa were close before the stress treatment, while the stress treatment had an obvious influence on the above indexes (Figure $6 \mathrm{E}$ ). $\mathrm{H}_{2} \mathrm{O}_{2}$, SOD, GSH, POD, Vc, and CAT levels are often used to reflect the extent of damage to plant cells, and the determination of these physiological indexes can help us to compare the response ability of the two quinoa to nitrogen deficiency stress (Figure 6F, Table S14). The results showed that the content of $\mathrm{H}_{2} \mathrm{O}_{2}$ was upregulated in both root systems and more significantly in LF quinoa after stress treatment; the content change of POD and CAT is similar to that of $\mathrm{H}_{2} \mathrm{O}_{2}$. Notably, both SOD and GSH levels in the stress-treated LF quinoa roots were significantly upregulated and downregulated, but this change was not significant in the SF quinoa roots. Moreover, the changing trend of Vc content in root system of two quinoa was the opposite in the root system of SF quinoa, Vc content was significantly upregulated after stress, which may help to alleviate oxidative damage caused by stress. PCA analysis of stress injury indicators showed that there were some differences in metabolites content in the roots of the two quinoa before stress, but the difference was more obvious after stress (Figure 6G).

The auxin and TCA cycle pathway genes in quinoa seeds may promote the root elongation of seedlings in a nitrogen starvation environment [49]. In order to explore the causes of differential phenotypes of two quinoa (LF and SF quinoa) under nitrogen starvation stress, we first mined the differentially expressed genes of auxin and TCA cycle pathways by RNA-seq data (Figure 6H, Table S15). We identified 9 (upregulation) and 10 (downregulation) differentially expressed genes in the IAA protein family, auxin biosynthesis and signal transduction pathways, and TCA cycle pathways (Figure $6 \mathrm{H}$, Table S15). On these bases, we further detected the expression levels of these pathway genes by the qPCR method (Figure 6I, Table S15). In total, 12 genes were found to have different expressions before and after nitrogen starvation treatments of the two quinoa (Figure 6I, Table S15). The riboflavin mono-oxygenase (YUC) genes in the auxin synthesis pathway were more significantly upregulated in LF quinoa, while the TRYPTOPHAN AMINOTRANSFERASES (TAA) genes were significantly downregulated in SF quinoa; the expression of these three genes was not significantly different before and after treatment with LF quinoa. In the auxin signaling pathway, the TIR1 gene was upregulated in LF quinoa after stress treatment, and TIR2 was upregulated in both quinoas. Interestingly, the encoding gene of ubiquitin-proteasome $(U b P)$ was downregulated in LF quinoa, which is in strong contrast to its upregulation in quinoa. The expression level of $P D C, I D H$, and $O G D C$ in both quinoas was upregulated in the tricarboxylic acid cycle pathway; $M D H$ was significantly upregulated in LF quinoa, in contrast to its significant downregulation in SF quinoa (Figure 6I, Table S15). The expression changes of OGDC (AUR62028926) and IAA (AUR62011942) in SF quinoa root after nitrogen starvation treatment are most significant $(p<0.001)$, respectively acting as key hubs in auxin signal and carbon-nitrogen metabolism balance, which may potentially promote root elongation of quinoa in a nitrogen-deficient environment (Figure 6I, Table S15). The above conclusions help us to understand the response mechanism of two quinoa seedlings in response to environmental nutritional deficiency and provide candidate genes for breeding varieties with tolerance to nitrogenstarvation soil. 

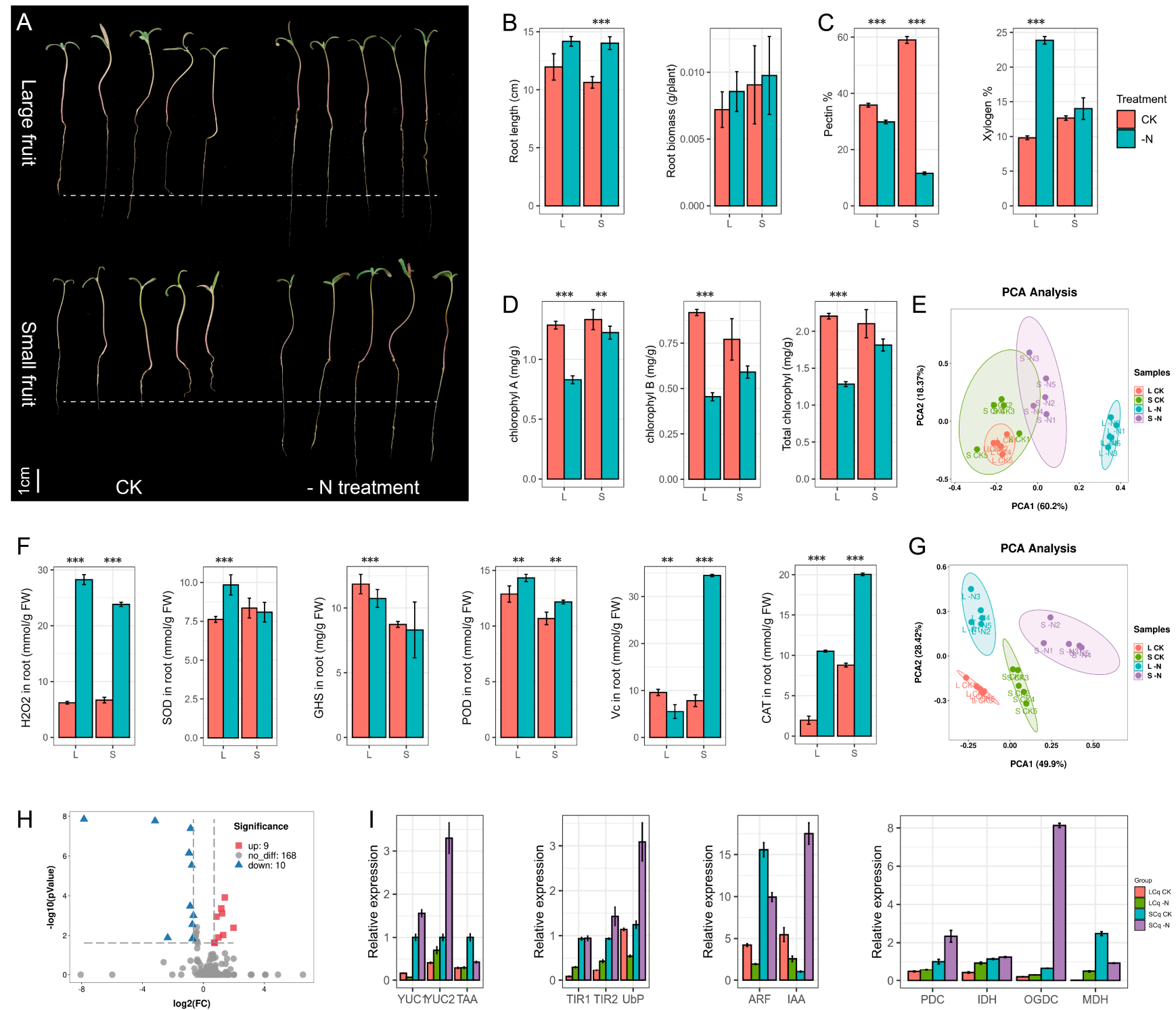

Figure 6. Physiological changes and gene expression differences of two quinoa seedlings in nitrogen-deficient environment. (A). Large-fruit and small-fruit quinoa seedlings under nitrogen deficiency stress. The first row was the big quinoa seedlings; the second row was the small quinoa seedlings; the left side and the right side were the control group and the experimental group, respectively; (B-D). Changes of physiological indexes of two quinoa seedlings under different treatments; ${ }^{* *}$ statistical significance for $p<0.05$; ${ }^{* * *}$ statistical significance for $p<0.001$; (E). Principal component (PCA) analysis of physiological index data of two materials under two treatments. The points of red, green, blue, and purple represent the control group of LF quinoa, the control group of SF quinoa, the experimental group of LF quinoa, and the experimental group of SF quinoa. Five biological repeats in each group (F). Response to stress responses of two quinoa varieties under different treatments (G). Principal component (PCA) analysis of physiological index data of two materials under two treatments. (H). Auxin biosynthesis, auxin signal transduction, and differential expression genes of TCA cycle pathway in two quinoa seeds. The volcanic map is generated at a Foldchange $>2, p$ value $<0.05$ threshold setting. Red and blue dots indicate genes that are significantly upregulated and downregulated in LF quinoa seeds, respectively; (I). Relative expression of genes CqIAAs, auxin pathway, and TCA pathway in different treatments of two quinoa. The genes used for qRT-PCR detection met the following conditions: the expression of quinoa seeds was significantly different and derived from the auxin pathway or TCA cycle pathway. 


\section{Discussion}

4.1. AUX/IAA Family May Originated after Plant Terrestrialization, Influenced by WGD Events, Formed Robust Networks with Auxin Signaling and Energy Metabolic Genes in Quinoa

The biosynthesis origin and signal transduction of auxin, as core components in the regulation of growth and development of plants, have long been a focus of both plant genome evolution and physiological research [50,51]. A large number of reports have been made on the transcriptional regulation mechanism of auxin signaling pathway in recent years, but they mainly focus on auxin response factor (ARF). Clear evolutionary biological evidence showed that the ARF family originated from early aquatic algae; in particular, the early origin and diversification of ARF in charophytes was recently delineated [52]. However, the auxin-reactive protein (AUX/IAA) family, which acts as an ARF interaction factor, also plays an irreplaceable role in the regulation of the auxin pathway but has little to report in the origin, evolution, and function of the family in depth. In this study, we compared almost all currently published aquatic algal genomes with the conservative structure of the AUX/IAA family, but without finding targets with significant structural consistency. This coincides with the findings of a recent report that genes encoding auxin signal transduction elements AUX/IAA, SAUR, and GH3 are missing in the genomes of Mesostigma viride and Chlorokybus atmophyticus [53]. Typical AUX/IAA family member was found in representative mosses, although they were small numerous and less structurally conserved. Identification of orthologous genes revealed that AUX/IAA families in most higher plants represented by Arabidopsis shared their original ancestors with bryophytes (Figure 1A-C). The evolution pattern of AUX/IAA is more conservative in higher plants, and the sequences of orthologous proteins in most plants are highly similar. In domesticated crops represented by quinoa, except for a few genes (such as AUR62011942) with a relatively fast evolution rate, most AUX/IAAs have conserved evolution patterns by strong purification selection (Figure 1G-K and Figure 2). After plant landification, AUX/IAA family expands and may be strongly influenced by several whole-genome duplication (WGD) events shared by angiosperms (Figure 3E,F). A lack of a strong synteny relationship between quinoa CqIAAs and AUX/IAAs in other species may suggest that the family's evolutionary pattern in quinoa is relatively independent (Figure 3D).

Most angiosperms have experienced major extinction events in history, such as the third great extinction in the Cretaceous. A large proportion of these species are thought to have escaped extinction destiny through the genome duplication strategy [54]. Due to the more reservations of stress-responsive genes in their different subgenomes, polyploid plants have been speculated to present a stronger ability to survive in the extreme ecological environment [55]. Polyploidized genes often form complex transcriptional regulatory networks to maintain plant homeostasis in response to extreme environments [56]. This study found that the IAA family of polyploid crops represented by quinoa expanded mainly through polyploidization events, and the CqIAAs retained in the quinoa tetraploid genome formed a robust regulatory network with various complex metabolism pathway genes in root. However, there is no strong correlation between CqIAAs and the regulatory network of metabolic pathways in the other four tissues (stem, leaf, flower, and fruit) (Figure 4I). Comparative transcriptome analysis showed that the robustness of the regulatory network involved by CqIAAs is different in the seed of two different quinoa (Figure 5), which may cause heterogeneity in energy metabolism and nutrient flow in the germination stage. The above conclusions suggest that the transcription factors family related to growth and development from the quinoa genome does form a closer metabolic regulatory network after polyploidization, which promotes its long-term domestication and adaptive evolution in extreme agroecological environments. Phylogenetic analysis of cross-species from aquatic lower plants to terrestrial angiosperms and multipathway regulatory network mining helps us to have a deeper understanding of the evolutionary origin and functional innovation of the AUX/IAA family. 
4.2. Root Elongation and Stress Response of Quinoa Seedlings in Nitrogen Starvation Are Helpful for Alleviating Nutritional Deficiency, and Small-Fruit Quinoa Has Better Potential Adaptability in Poor Agricultural Environmental

Nitrogen has diverse and irreplaceable necessities in various stages of plant growth and development. By being the building blocks for participants in basic biological processes such as nucleic acids, proteins, and chlorophyll, they provide important components for cell metabolic activity and structure [57]. In addition, nitrogen as a raw material for tryptophan metabolism plays a role in plant hormone-mediated growth and development regulation [58]. Nitrogen homeostasis in plant cells strongly depends on mitochondrialmediated carbon-nitrogen transformation equilibrium [49]. More and more reports suggest that mitochondrial function and auxin are closely interconnected: mitochondrial metabolic activity regulates auxin signaling, which promotes organogenesis and morphogenesis in plants [59]; in Arabidopsis thaliana, the retrograde signal of mitochondria can be connected to the polar distribution of auxin, which changes the phenotype of plant roots $[60,61]$; in rice, mutations in mitochondrial structural genes affect auxin biosynthesis and signaling pathways, thus altering the morphological structure of plant vascular bundles [62]. The effect of mitochondria on the auxin pathway is in fact coupled through TCA cycles. Amino acids are important intermediates substrates that transition from carbon metabolism to nitrogen metabolism in the TCA cycle and can enter the tryptophan-dependent IAA biosynthesis pathway [63,64]. In addition, studies have shown that plants can interact with microorganisms to mediate salt stress response via ACC-deaminase and auxin activity [65]. The synthesis of phenol and flavonoid secondary metabolites and the change of endogenous hormones such as abscisic acid and auxin also promoted plant growth and development and stress resistance [66]. This further suggests that metabolism balance plays an important role in crop resistance to environmental stress.

The transcriptional levels of such genes in the TCA cycle and auxin pathway were significantly different in the seeds of the two quinoa materials selected in this study, suggesting that the carbon-nitrogen metabolism of two quinoa species during germination may enter into different metabolic streams. A generally accepted view is that the seed germination stage is most easily disturbed by environmental stress, and the stress responseability of seedlings directly determines the survival rate of plants $[67,68]$. Our results of the experiment are as expected that under nitrogen deficiency stress, the root of SF quinoa seedlings had obvious elongation, and chlorophyll, cell wall composition, and physiological indexes related to oxidative damage showed better characterization than LF quinoa seedlings (Figure 6A-G). qPCR detection confirmed that the expression of TCA and auxin-related genes were strongly induced in roots under nitrogen-deficient stress (Figure 6I). The above conclusions further confirm our inference that the AUX/IAA family in SF quinoa forms a robust regulatory network with TCA cycle and auxin pathway genes, thus enabling the carbon-nitrogen metabolism balance to be more actively deployed in the nitrogen deficiency environment, thus maintaining the physiological homeostasis in the barren environment. Noticeably, the rapidly evolving gene AUX62011942 found by evolution analysis not only has significantly different expression levels in LF quinoa seeds and SF quinoa but also shows a strong response when young roots of SF quinoa face nitrogen stress. Moreover, CqIAA was significantly upregulated under nitrogen stress in SF root, while $C q A R F$ was downregulated, in sharp contrast to the consistent downregulation of the two genes during the stress of LF root (Figure 6I). Phytohormone plays an important role in plants' resistance to environmental stress [69]. Herein, we present a possible mechanism model: before germination, SF quinoa seeds form a hormone and energy metabolism regulation network centered on IAA, and after germination in a nutrientdeficient environment, the complex regulation network affects auxin signal, thus inducing root elongation to seek more nitrogen sources. By coupling the energy metabolism pathway, the intracellular carbon-nitrogen metabolism was rearranged, which together maintained the homeostasis of seedlings in the nitrogen deficiency environment. The above inference needs to be supported by further experimental evidence. To sum up, it is a more efficient genetic and agricultural breeding strategy to excavate the dominant genes preserved by 
environmental selection at the evolutionary scale, thus providing valuable insights for understanding the evolutionary strategies of crops adapting to the complex agroecological environment in the course of domestication.

\section{Conclusions}

Our study examines the AUX/IAA family in 19 plants with agricultural and evolutionary importance, from aquatic algae to angiosperms. The results show that the AUX/IAA family originated after the territization of plants and expanded in angiosperms, which has a conservative evolutionary pattern and finds general conservation of function across species. We also emphasized AUX/IAAs' expansion in angiosperms is due to polyploidization events. We tapped the complex regulatory networks from quinoa transcriptome datasets, which found that AUX/IAA family members show interactions with auxin signaling and energy metabolism genes. More importantly, the stress response of two different quinoa materials under nitrogen starvation was different. CqIAAs, hormone signaling, and energy metabolism pathway genes in quinoa seedlings showed significant responses under nitrogen starvation stress, which provide guidance for understanding the adaptive mechanism of crop adaptation to poor agroecological environments.

Supplementary Materials: The following are available online at https://www.mdpi.com/article/ 10.3390 /agriculture11080780/s1, Table S1. Basic information on 495 IAA proteins identified from 17 plant genomes, Table S2. Orthogroup identification of multispecies IAA family proteins, Table S3. Prediction of cis-acting elements in promoter regions of 37 quinoa CqIAAs, Table S4. The synonymous (Ks) and nonsynonymous (Ka) substitutions rates of all CqIAAs, Table S5. Synteny IAA gene pairs in the genome of Chenopodium quinoa, Triticum aestivum and Brassica napus, Table S6. Synteny IAA gene pairs between Chenopodium quinoa and other species, Table S7. Identification of repeat types of IAAs in different species, Table S8. Organizational expression matrix, trend analysis of gene expression of 49 profiles and KEGG pathway enrichment analysis of three Gene profiles specifically expressed in roots, Table S9. Coexpression networks formed by CqIAAs in roots and other tissues, Table S10. GO enrichment analysis of target genes associated with IAA in the coexpression network, Table S11. Transcription level (FPKM) of 37 CQIAA in two quinoa species and morphological data of seeds in two quinoa species, Table S12. Transcription level (FPKM) of auxin biosynthesis, auxin signal transduction, and tricarboxylic acid cycle pathway genes, Table S13. Coexpression networks in LF and SF quinoa seeds formed by CqIAA and hormone and energy metabolic pathway genes, Table S14. Physiological indexes of two kinds of quinoa under nitrogen stress, Table S15. Gene expression of roots of two quinoa seedlings under nitrogen deficiency stress, Table S16. Primer sequence for qRT-PCR experiment.

Author Contributions: H.Y. designed and participated in the whole study and wrote the manuscript; S.W. analyzed and visualized plant genome data; Y.Y. carried out plant culture, physiological experiments, and data analysis; H.X. was involved in the comparative genome analysis of multiple species; G.W. was involved in metabolic pathways and regulatory network analysis; J.W., Y.H. and L.J. summarized and analyzed data generated from the experiments; H.C. provided primary guidance and platform support throughout the study and reviewed and approved the manuscript. All authors have read and agreed to the published version of the manuscript.

Funding: This research was supported by a project of Onentrepreneurship Training Program for College Students (202010626015) from Ministry of Education of PRC, and a support program of Function study of FtbHLH transcription factor regulating Tartary buckwheat fruit dehiscence (2021YFH0086) from Sichuan province science and technology. Funds were used for the design of the study and collection, analysis, and interpretation of data and in writing the manuscript, as well as in the open-access payment.

Institutional Review Board Statement: Not applicable.

Informed Consent Statement: Not applicable.

Data Availability Statement: The datasets supporting the conclusions of this article are included with in the article and its Supplementary Files. 
Acknowledgments: We thank all the colleagues in our laboratory for providing useful discussions and technical assistance. All authors read and approved the final manuscript. We would also like to thank Mengyuan Lv, School of Life Sciences and Technology, University of Electronic Science and Technology of China, for providing experimental support.

Conflicts of Interest: The authors declare that they have no competing interests.

$\begin{array}{ll}\text { Abbreviations } \\ \text { Arabidopsis } & \text { Arabidopsis thaliana } \\ \text { AtIAAs } & \text { Arabidopsis thaliana IAAs } \\ \text { CqIAAs } & \text { Chenopodium quinoa IAAs } \\ \text { GO } & \text { Gene ontology } \\ \text { IAAs } & \text { AUX/IAA genes } \\ \text { LF quinoa } & \text { Large-fruit quinoa } \\ \text { MCScanX } & \text { Multiple Collinearity Scan toolkit } \\ \text { SF quinoa } & \text { Small-fruit quinoa } \\ \text { WGD } & \text { Whole-genome duplications }\end{array}$

\section{References}

1. Fürst-Jansen, J.; De Vries, S.; de Vries, J. Evo-physio: On stress responses and the earliest land plants. J. Exp. Bot. 2020, 71, 3254-3269. [CrossRef] [PubMed]

2. Han, X.; Chang, X.; Zhang, Z.; Chen, H.; He, H.; Zhong, B.; Deng, X. Origin and Evolution of Core Components Responsible for Monitoring Light Environment Changes during Plant Terrestrialization. Mol. Plant 2019, 12, 847-862. [CrossRef] [PubMed]

3. Jianguo, G. Tracking the evolutionary innovations of plant terrestrialization. Gene 2020, 769, 145203.

4. Abel, S.; Theologis, A. Early genes and auxin action. Plant Physiol. 1996, 111, 9-17. [CrossRef]

5. Weijers, D.; Wagner, D. Transcriptional Responses to the Auxin Hormone. Annu. Rev. Plant Biol. 2016, 67, 539-574. [CrossRef] [PubMed]

6. Gray, W.; Kepinski, S.; Rouse, D.; Leyser, O.; Estelle, M. Auxin regulates SCF-dependent degradation of AUX/IAA proteins Nature 2001, 414, 271-276. [CrossRef]

7. Zenser, N.; Ellsmore, A.; Leasure, C.; Callis, J. Auxin modulates the degradation rate of Aux/IAA proteins. Proc. Natl. Acad. Sci. USA 2001, 98, 11795-11800. [CrossRef]

8. Yamaguchi, N.; Wu, M.; Winter, C.; Berns, M.; Nole-Wilson, S.; Yamaguchi, A.; Coupland, G.; Krizek, B.; Wagner, D. A molecular framework for auxin-mediated initiation of flower primordia. Dev. Cell 2013, 24, 271-282. [CrossRef]

9. Roosjen, M.; Paque, S.; Weijers, D. Auxin Response Factors: Output control in auxin biology. J. Exp. Bot. 2017, 69, 179-188. [CrossRef]

10. Mutte, S.; Kato, H.; Rothfels, C.; Melkonian, M.; Wong, G.; Weijers, D. Origin and evolution of the nuclear auxin response system. Elife 2018, 7, e33399. [CrossRef]

11. Flores-Sandoval, E.; Eklund, D.M.; Hong, S.-F.; Alvarez, J.; Fisher, T.; Lampugnani, E.; Golz, J.; Vazquez-Lobo, A.; Dierschke, T.; Lin, S.-S.; et al. Class C ARFs evolved before the origin of land plants and antagonize differentiation and developmental transitions in Marchantia polymorpha. New Phytol. 2018, 218, 1612-1630. [CrossRef]

12. Bazile, D.; Jacobsen, S.-E.; Verniau, A. The Global Expansion of Quinoa: Trends and Limits (Mini Review). Front. Plant Sci. 2016, 7, 622. [CrossRef]

13. Jacobsen, S.E. The Worldwide Potential for Quinoa (Chenopodium quinoa Willd.). Food Rev. Int. 2003, 19, 167-177. [CrossRef]

14. Repo-Carrasco-Valencia, R.; Espinoza, C.; Jacobsen, S.-E. Nutritional Value and Use of the Andean Crops Quinoa (Chenopodium quinoa) and Kañiwa (Chenopodium pallidicaule). Food Rev. Int. Food Rev. Int. 2003, 19, 179-189. [CrossRef]

15. Jarvis, D.; Ho, Y.S.; Lightfoot, D.; Schmöckel, S.; Li, B.; Borm, T.; Ohyanagi, H.; Mineta, K.; Michell, C.; Saber, N.; et al. The genome of Chenopodium quinoa. Nature 2017, 542, 1-6. [CrossRef] [PubMed]

16. Pevsner, J. Basic Local Alignment Search Tool (BLAST). Mol. Biol. 2009, 215, 100-138.

17. Xu, L.; Dong, Z.; Fang, L.; Luo, Y.; Wei, Z.; Guo, H.; Zhang, G.; Gu, Y.; Coleman-Derr, D.; Xia, Q.; et al. OrthoVenn2: A web server for whole-genome comparison and annotation of orthologous clusters across multiple species. Nucleic Acids Res. 2019, 47, W52-W58. [CrossRef] [PubMed]

18. Bailey, T.; Bodén, M.; Buske, F.; Frith, M.; Grant, C.; Clementi, L.; Ren, J.; Li, W.; Noble, W. MEME SUITE: Tools for motif discovery and searching. Nucleic Acids Res. 2009, 37, W202-8. [CrossRef]

19. Chen, C.; Chen, H.; Zhang, Y.; Thomas, H.; Frank, M.; He, Y.; Xia, R. TBtools: An Integrative Toolkit Developed for Interactive Analyses of Big Biological Data. Mol. Plant 2020,13, 1194-1202. [CrossRef] [PubMed]

20. Liu, M.; Ma, Z.; Zheng, T.; Sun, W.; Zhang, Y.; Jin, W.; Zhan, J.; Cai, Y.; Tang, Y.; Wu, Q.; et al. Insights into the correlation between Physiological changes in and seed development of tartary buckwheat (Fagopyrum tataricum Gaertn.). BMC Genom. 2018, 19, 648. [CrossRef] 
21. Paterson, A.H. MCScanX: A toolkit for detection and evolutionary analysis of gene synteny and collinearity. Nucleic Acids Res. 2012, 40, e49.

22. Song, X.; Li, Y.; Hou, X. Genome-wide analysis of the AP2/ERF transcription factor superfamily in Chinese cabbage (Brassica rapa ssp. pekinensis). BMC Genom. 2013, 14, 573. [CrossRef] [PubMed]

23. Naggert, J.K. Defective carbohydrate metabolism in mice homozygous for the tubby mutation. Physiol. Genom. 2006, 27, 131-140.

24. Wang, X. Hierarchically Aligning 10 Legume Genomes Establishes a Family-Level Genomics Platform. Plant Physiol. 2017, 174, 284-300. [CrossRef]

25. Robles, M. Blast2GO: A universal tool for annotation, visualization and analysis in functional genomics research. Bioinformatics 2005, 21, 3674-3676.

26. Xie, C.; Huang, J.; Ding, Y.; Wu, J.; Dong, S.; Kong, L.; Gao, G.; Li, C.-Y.; Wei, L. KOBAS 2.0: A web server for annotation and identification of enriched pathways and diseases. Nucleic Acids Res. 2011, 39, W316-W322. [CrossRef]

27. Ernst, J.; Bar-Joseph, Z. STEM: A tool for the analysis of short time series gene expression data. BMC Bioinform. 2006, 7, 191. [CrossRef] [PubMed]

28. Wang, D.; Zhang, Y.; Zhang, Z.; Zhu, J.; Yu, J. KaKs_Calculator 2.0: A Toolkit Incorporating Gamma-Series Methods and Sliding Window Strategies. Genom. Proteom. Bioinform. 2010, 8, 77-80. [CrossRef]

29. Liu, M.; Sun, W.; Li, C.; Yu, G.; Li, J.; Wang, Y.; Wang, X. A multilayered cross-species analysis of GRAS transcription factors uncovered their functional networks in plant adaptation to the environment. J. Adv. Res. 2020, 29, 191-205. [CrossRef] [PubMed]

30. Liu, M.; Sun, W.; Ma, Z.; Yu, G.; Li, J.; Wang, Y.; Wang, X. Comprehensive multiomics analysis reveals key roles of NACs in plant growth and development and its environmental adaption mechanism by regulating metabolite pathways. Genomics 2020, 112, 4897-4911. [CrossRef] [PubMed]

31. Zhang, Z.-W.; Dong, Y.-Y.; Feng, L.-Y.; Deng, Z.-L.; Xu, Q.; Tao, Q.; Wang, C.-Q.; Chen, Y.-E.; Yuan, M.; Yuan, S. Selenium Enhances Cadmium Accumulation Capability in Two Mustard Family Species_Brassica napus and B. juncea. Plants 2020, 9, 904. [CrossRef]

32. Ruiz Carrasco, K.; Antognoni, F.; Coulibaly, A.; Lizardi, S.; Covarrubias, A.; Martínez, E.; Molina-Montenegro, M.; Biondi, S.; Zurita-Silva, A. Variation in salinity tolerance of four lowland genotypes of quinoa (Chenopodium quinoa Willd.) as assessed by growth, physiological traits, and sodium transporter gene expression. Plant Physiol. Biochem. PPB Société Française De Physiol. Végétale 2011, 49, 1333-1341. [CrossRef]

33. Livak, K.J.; Schmittgen, T.D. Analysis of relative gene expression data using real-time quantitative PCR and the 2(-Delta Delta C(T)) Method. Methods 2012, 25, 402-408. [CrossRef]

34. Jiao, Y.; Wickett, N.; Ayyampalayam, S.; Chanderbali, A.; Landherr, L.; Ralph, P.; Tomsho, L.; Hu, Y.; Liang, H.; Soltis, P.; et al. Ancestral polyploidy in seed plants and angiosperms. Nature 2011, 473, 97-100. [CrossRef]

35. Aguilar, P.; Jacobsen, S. Cultivation of Quinoa on the Peruvian Altiplano. Food Reviews International. 2003, 19, 31-41. [CrossRef]

36. Freeling, M. Bias in Plant Gene Content Following Different Sorts of Duplication: Tandem, Whole-Genome, Segmental, or by Transposition. Annu. Rev. Plant Biol. 2009, 60, 433-453. [CrossRef] [PubMed]

37. Schilling, S.; Kennedy, A.; Pan, S.; Jermiin, L.; Melzer, R. Genome-wide analysis of MIKC-type MADS-box genes in wheat: Pervasive duplications, functional conservation and putative neofunctionalization. New Phytol. 2019, 225, 511-529. [CrossRef] [PubMed]

38. Xu, J.-H.; Messing, J. Diverged Copies of the Seed Regulatory Opaque-2 Gene by a Segmental Duplication in the Progenitor Genome of Rice, Sorghum, and Maize. Mol. Plant 2008, 1, 760-769. [CrossRef] [PubMed]

39. Chapman, B.; Bowers, J.; Schulze, S.; Paterson, A. A comparative phylogenetic approach for dating whole genome duplication events. Bioinformatics 2004, 20, 180-185. [CrossRef] [PubMed]

40. Van de Peer, Y.; Maere, S.; Meyer, A. The evolutionary significance of ancient genome duplications. Nat. Rev. Genet. 2009, 10, 725-732. [CrossRef]

41. Matosevich, R.; Cohen, I.; Gil-Yarom, N.; Modrego, A.; Friedlander-Shani, L.; Verna, C.; Scarpella, E.; Efroni, I. Local auxin biosynthesis is required for root regeneration after wounding. Nat. Plants 2020, 6, 1020-1030. [CrossRef]

42. Motte, H.; Vanneste, S.; Beeckman, T. Molecular and Environmental Regulation of Root Development. Annu. Rev. Plant Biol. 2019, 70, 465-488. [CrossRef]

43. Xiao, G.; Zhang, Y. Adaptive Growth: Shaping Auxin-Mediated Root System Architecture. Trends Plant Sci. 2019, 25, 121-123. [CrossRef] [PubMed]

44. Holdsworth, M.; Bentsink, L.; Soppe, W. Molecular networks regulating Arabidopsis seed maturation, after-ripening, dormancy and germination. New Phytol. 2008, 179, 33-54. [CrossRef] [PubMed]

45. Nietzel, T.; Mostertz, J.; Ruberti, C.; Née, G.; Fuchs, P.; Wagner, S.; Moseler, A.; Müller-Schüssele, S.; Benamar, A.; Poschet, G.; et al. Redox-mediated kick-start of mitochondrial energy metabolism drives resource-efficient seed germination. Proc. Natl. Acad. Sci. USA 2020, 117, 741-751. [CrossRef] [PubMed]

46. Rosental, L.; Nonogaki, H.; Fait, A. Activation and regulation of primary metabolism during seed germination. Seed Sci. Res. 2014, 24, 1-15. [CrossRef]

47. Zhang, Y.; Swart, C.; Alseekh, S.; Scossa, F.; Jiang, L.; Obata, T.; Graf, A.; Fernie, A. The Extra-Pathway Interactome of the TCA Cycle: Expected and Unexpected Metabolic Interactions. Plant Physiol. 2018, 177, 966-979. [CrossRef]

48. Gautrat, P.; Laffont, C.; Frugier, F.; Ruffel, S. Nitrogen Systemic Signaling: From Symbiotic Nodulation to Root Acquisition. Trends Plant Sci. 2020, 26, 392-406. [CrossRef] 
49. Ryan, D.; Frezza, C.; O’Neill, L. TCA cycle signalling and the evolution of eukaryotes. Curr. Opin. Biotechnol. 2021, 68, 72-88. [CrossRef]

50. Chapman, E.; Estelle, M. Mechanism of Auxin-Regulated Gene Expression in Plants. Annu. Rev. Genet. 2009, 43, 265-285. [CrossRef]

51. Kieffer, M.; Neve, J.; Kepinski, S. Defining auxin response contexts in plant development. Curr. Opin. Plant Biol. 2009, 13, 12-20. [CrossRef]

52. Gao, B.; Wang, L.; Oliver, M.; Chen, M.-X.; Zhang, J. Evolution of Auxin Response Factors in plants characterized by phylogenomic synteny network analyses. BioRxiv 2019, 603175, 1-22.

53. Wang, S.; Li, L.; Li, H.; Sahu, S.K.; Wang, H.; Xu, Y.; Xian, W.; Song, B.; Liang, H.; Cheng, S.; et al. Genomes of early-diverging streptophyte algae shed light on plant terrestrialization. Nat. Plants 2020, 6, 95-106. [CrossRef]

54. Mabry, M.; Brose, J.; Blischak, P.; Sutherland, B.; Dismukes, W.; Bottoms, C.; Edger, P.; Washburn, J.; An, H.; Hall, J.; et al. Phylogeny and Multiple Independent Whole-Genome Duplication Events in the Brassicales. Am. J. Bot. 2020, 107, $1148-1164$. [CrossRef] [PubMed]

55. Prentis, P.; Wilson, J.; Dormontt, E.; Richardson, D.; Lowe, A. Adaptive evolution in invasive species. Trends Plant Sci. 2008, 13, 288-294. [CrossRef]

56. Song, X.; Wang, J.; Sun, P.; Ma, X.; Yang, Q.; Hu, J.; Sun, S.; Li, Y.; Yu, J.; Feng, S.; et al. BrassicaceaePreferential gene retention increases the robustness of cold regulation in and other plants after polyploidization. Hortic. Res. 2020, 7, 20. [CrossRef] [PubMed]

57. Tegeder, M. Transporters for amino acids in plant cells: Some functions and many unknowns. Curr. Opin. Plant Biol. 2012, 15, 315-321. [CrossRef] [PubMed]

58. Hayashi, K. Chemical Biology in Auxin Research. Cold Spring Harb. Perspect. Biol. 2021, 13, a040105. [CrossRef] [PubMed]

59. Kerchev, P.; Clercq, I.; Denecker, J.; Mühlenbock, P.; Kumpf, R.; Nguyen, L.; Audenaert, D.; Dejonghe, W.; Breusegem, F. Mitochondrial perturbation negatively affects auxin signaling. Mol. Plant. 2014, 7, 1138-1150. [CrossRef]

60. Ivanova, A.; Law, S.; Narsai, R.; Duncan, O.; Lee, J.; Zhang, B.; Van Aken, O.; Radomiljac, J.; van der Merwe, M.; Yi, K.; et al. A Functional Antagonistic Relationship between Auxin and Mitochondrial Retrograde Signaling Regulates Alternative Oxidase1a Expression in Arabidopsis. Plant Physiol. 2014, 165, 1233-1254. [CrossRef]

61. Wang, X.; Auwerx, J. Systems Phytohormone Responses to Mitochondrial Proteotoxic Stress. Mol. Cell 2017, 68, 540-551.e5. [CrossRef]

62. Zhang, L.; Feng, P.; Deng, Y.; Yin, W.; Wan, Y.; Lei, T.; He, G.; Wang, N. Decreased Vascular Bundle 1 affects mitochondrial and plant development in rice. Rice 2021, 14, 13. [CrossRef]

63. Cogliati, S.; Frezza, C.; Soriano, M.; Varanita, T.; Quintana-Cabrera, R.; Corrado, M.; Cipolat, S.; Costa, V.; Casarin, A.; Gomes, L.; et al. Mitochondrial cristae shape determines respiratory chain supercomplexes assembly and respiratory efficiency. Cell 2013, 155, 160-171. [CrossRef] [PubMed]

64. Benstein, R.; Ludewig, K.; Wulfert, S.; Wittek, S.; Gigolashvili, T.; Frerigmann, H.; Gierth, M.; Flügge, U.-I.; Krueger, S. Arabidopsis Phosphoglycerate Dehydrogenase1 of the Phosphoserine Pathway Is Essential for Development and Required for Ammonium Assimilation and Tryptophan Biosynthesis. Plant Cell 2013, 25, 5011-5029. [CrossRef]

65. Yang, A.; Akhtar, S.S.; Iqbal, S.; Amjad, M.; Naveed, M.; Zahir, Z.A.; Jacobsen, S.E. Enhancing salt tolerance in quinoa by halotolerant bacterial inoculation. Funct. Plant Biol. 2016, 43, 632-642. [CrossRef]

66. Sheteiwy, M.S.; AbdElgawad, H.; Xiong, Y.C.; Macovei, A.; Brestic, M.; Skalicky, M.; Shaghaleh, H.; Hamoud, Y.A.; El-Sawah, A.M. Inoculation with Bacillus amyloliquefaciens and mycorrhiza confers tolerance to drought stress and improve seed yield and quality of soybean plant. Physiol. Plant. 2021, 172, 2153-2169. [CrossRef] [PubMed]

67. Hajihashemi, S.; Skalicky, M.; Brestic, M.; Pavla, V. Cross-talk between nitric oxide, hydrogen peroxide and calcium in salt-stressed Chenopodium quinoa Willd. At seed germination stage. Plant Physiol. Biochem. 2020, 154, 657-664. [CrossRef] [PubMed]

68. Sun, W.; Yu, H.; Ma, Z.; Yuan, Y.; Wang, S.; Yan, J.; Xu, X.; Chen, H. Molecular Evolution and Local Root Heterogeneous Expression of the Chenopodium quinoa ARF Genes Provide Insights into the Adaptive Domestication of Crops in Complex Environments. J. Mol. Evol. 2021, 89, 287-301. [CrossRef]

69. Sharma, A.; Sidhu, G.P.S.; Araniti, F.; Bali, A.S.; Shahzad, B.; Tripathi, D.K.; Brestic, M.; Skalicky, M.; Landi, M. The Role of Salicylic Acid in Plants Exposed to Heavy Metals. Molecules 2020, 25, 540. [CrossRef] 